\title{
Genetic epidemiology of autoinflammatory disease variants in Indian population from 1029 whole genomes
}

\begin{abstract}
Abhinav Jain 1,2, Rahul C. Bhoyar ${ }^{1}$, Kavita Pandhare1 , Anushree Mishra', Disha Sharma' , Mohamed Imran ${ }^{1,2}$, Vigneshwar Senthivel 1,2, Mohit Kumar Divakar ${ }^{1,2}$, Mercy Rophina ${ }^{1,2}$, Bani Jolly ${ }^{1,2}$, Arushi Batra ${ }^{1,2}$, Sumit Sharma ${ }^{1}$, Sanjay Siwach', Arun G. Jadhao ${ }^{3}$, Nikhil V. Palande ${ }^{4}$, Ganga Nath Jha ${ }^{5}$, Nishat Ashrafi ${ }^{5}$, Prashant Kumar Mishra ${ }^{6}$, Vidhya A.K. ${ }^{7}$, Suman Jain ${ }^{8}$, Debasis Dash ${ }^{1,2}$, Nachimuthu Senthil Kumar ${ }^{9}$, Andrew Vanlallawma ${ }^{9}$, Ranjan Jyoti Sarma ${ }^{9}$, Lalchhandama Chhakchhuak ${ }^{10}$, Shantaraman Kalyanaraman ${ }^{11}$, Radha Mahadevan ${ }^{11}$, Sunitha Kandasamy ${ }^{11}$, Pabitha B. M, ${ }^{11}$, Raskin Erusan Rajagopal ${ }^{11}$, Ezhil Ramya J. ${ }^{11}$, Nirmala Devi P. ${ }^{11}$, Anjali Bajaj ${ }^{1,2}$, Vishu Gupta 1,2, Samatha Mathew ${ }^{1,2}$, Sangam Goswami 1,2, Mohit Mangla 1,2, Savinitha Prakash', Kandarp Joshi', Meyakumla', Sreedevi S. ${ }^{12}$, Devarshi Gajjar ${ }^{13}$, Ronibala Soraisham ${ }^{14}$, Rohit Yadav ${ }^{1,2}$, Yumnam Silla Devi ${ }^{15}$, Aayush Gupta ${ }^{16}$, Mitali Mukerji ${ }^{1,2}$, Sivaprakash Ramalingam,2, Binukumar B. K. ${ }^{1,2}$, Vinod Scaria ${ }^{1,2^{*}}$ (I) and Sridhar Sivasubbu 1, $^{1 *}$
\end{abstract}

\begin{abstract}
Background: Autoinflammatory disorders are the group of inherited inflammatory disorders caused due to the genetic defect in the genes that regulates innate immune systems. These have been clinically characterized based on the duration and occurrence of unprovoked fever, skin rash, and patient's ancestry. There are several autoinflammatory disorders that are found to be prevalent in a specific population and whose disease genetic epidemiology within the population has been well understood. However, India has a limited number of genetic studies reported for autoinflammatory disorders till date. The whole genome sequencing and analysis of 1029 Indian individuals performed under the IndiGen project persuaded us to perform the genetic epidemiology of the autoinflammatory disorders in India.
\end{abstract}

Results: We have systematically annotated the genetic variants of 56 genes implicated in autoinflammatory disorder. These genetic variants were reclassified into five categories (i.e., pathogenic, likely pathogenic, benign, likely benign, and variant of uncertain significance (VUS)) according to the American College of Medical Genetics and Association of Molecular pathology (ACMG-AMP) guidelines. Our analysis revealed 20 pathogenic and likely pathogenic variants with significant differences in the allele frequency compared with the global population. We also found six causal founder variants in the IndiGen dataset belonging to different ancestry. We have performed haplotype prediction analysis for founder mutations haplotype that reveals the admixture of the South Asian population with other populations. The cumulative carrier frequency of the autoinflammatory disorder in India was found to be $3.5 \%$ which is much higher than reported.

\footnotetext{
*Correspondence: vinods@igib.in; sridhar@igib.in

${ }^{2}$ Academy of Scientific and Innovative Research (AcSIR), Ghaziabad, Uttar

Pradesh 201002, India

Full list of author information is available at the end of the article
}

(c) The Author(s) 2021. Open Access This article is licensed under a Creative Commons Attribution 4.0 International License, which permits use, sharing, adaptation, distribution and reproduction in any medium or format, as long as you give appropriate credit to the original author(s) and the source, provide a link to the Creative Commons licence, and indicate if changes were made. The images or other third party material in this article are included in the article's Creative Commons licence, unless indicated otherwise in a credit line to the material. If material is not included in the article's Creative Commons licence and your intended use is not permitted by statutory regulation or exceeds the permitted use, you will need to obtain permission directly from the copyright holder. To view a copy of this licence, visit http://creativecommons.org/licenses/by/4.0/. 
Conclusion: With such frequency in the Indian population, there is a great need for awareness among clinicians as well as the general public regarding the autoinflammatory disorder. To the best of our knowledge, this is the first and most comprehensive population scale genetic epidemiological study being reported from India.

Keywords: Autoinflammatory disorder, Genetic epidemiology, American College of Medical Genetics and Genomics, Allele frequency, Haplotype ancestry

\section{Background}

Autoinflammatory disorders are the growing group of Mendelian disorders caused by genetic defects in the genes which regulate the innate immune system. These disorders have been characterized by recurrent episodes of fever, abdominal pain, skin rashes, arthritis, serositis, conjunctivitis, or cutaneous signs that lack specificity and therefore make diagnosis difficult $[1,2]$. These disorders are clinically diagnosed based on the age of onset, duration of fever and flares, type of rash, family history, and patient's ancestry [3]. Autoinflammatory disorders have a strong genetic background, multiple new genes or variants have been recently discovered [4-7]. The recent advancement in next-generation sequencing has resulted in the identification of more than 30 new genes associated with autoinflammatory disorders $[8,9]$. Classically, most monogenic autoinflammatory disorders follow autosomal dominant and autosomal recessive modes of inheritance. Molecular diagnosis along with clinical criteria is essential for confirmation of the disease as well as it aids in discovering new disease [10]. Even autoinflammatory disorders manifest heterogeneity in phenotype-genotype correlation (i.e., mutation in the same gene can have varying severity and clinical manifestation and mutation in different genes can result in similar clinical characteristics) [11-13].

Identification of the causal variants in the genes responsible for autoinflammatory disorder mainly uses clinical or whole exome sequencing. However, $60 \%$ of patients suspected with autoinflammatory disorder remain molecularly undiagnosed using these sequencing technologies [14]. The undiagnosis could be due to the inability of the sequencing platforms in calling structural variants (SVs) [15], missing non-coding variants [16] as well as inadequate coverage of the coding region [17], and high-quality SNVs [18]. However the whole genome sequencing (WGS) has the ability to identify variants in difficult-to-diagnose cases. Recently, our group has identified $\sim 5 \mathrm{~Kb}$ deletion in the primary immunodeficiency disorder (PID) patients that could not be identified using whole exome sequencing [19]. Also, Thaventhiran et al. has identified eight SVs by performing the WGS of 1318 patients affected with PID, that could be missed using the WES [20]. WGS has also been implemented at the population scale for autoinflammatory disorders to comprehend the genetic epidemiology of the Qatari population [21]. Even though India is a hotspot for the genetic disorder due to the prevalent practice of endogamy and consanguineous marriages, migration, and large population size, the genetic epidemiology of autoinflammatory disorders in the country has not been studied much except for a few reports [22].

The whole-genome sequence data for cosmopolitan Indian populations encompassing 1029 individuals as part of the IndiGen programme [23] motivated us to estimate the genetic epidemiology of autoinflammatory disorders. In the present analysis, we have performed extensive data mining as well as integrative analysis to evaluate the pathogenicity of the variant according to ACMG-AMP guidelines. We have also further analyzed the variant prevalence in India compared to the global population. This is the first most comprehensive genetic epidemiology performed for autoinflammatory disorders in India.

\section{Methods \\ IndiGen dataset and variant annotation}

A total of 59,646,267 genetic variants including single-nucleotide variants and Indels from the IndiGen dataset were considered for the analysis. The data was derived from whole genome sequencing of 1029 cosmopolitan healthy Indians with a well-written informed consent obtained [23]. These are self-declared healthy individuals selected from different states of India without any provisional diagnosis of autoinflammatory disorder. It includes 495 males and 534 females with mean ages of 41.35 and 32.96 years, respectively [23]. These variants obtained were annotated using a tool called ANNOVAR (v. 2018-04-06) [24]. ANNOVAR provides annotations from multiple databases that include RefGene [25] and dbSNP (avsnp150) [26] that provide detailed information about the variant, dbNSFP35a that comprises data of multiple pathogenicity prediction tools [27]; global population databases (gnomAD V3 [28], 1000 Genome Project [29], Esp6500 [30], and Greater Middle East (GME)) provide allele frequency of different ancestry [31]. Finally, variant clinical 
significance of variants was was retrieved from ClinVar (ver 2020-01-13) database [32].

\section{IndiGen variant filtering}

Out of the total IndiGen variants, we have extracted variants from 56 genes associated with the 47 autoinflammatory disorders. These autoinflammatory disorder genes were selected by the experts of the International Union of Immunological Societies (IUIS) [33] and Infever as tabulated in Table 1. Further, those variants that are mapped to the exonic (except synonymous) and splicing region or those that were pathogenic and likely pathogenic variants at the other genomic positions as per ClinVar were prioritized. Also, variants whose allele frequency is less than 0.05 in the global population datasets (1000 genome project, gnomAD V3, and Esp6500) were considered for further analysis. As the variants with allele frequency greater than 0.05 were considered polymorphic and present in the large number of healthy individuals across different populations.

\section{Datasets of disease-associated genetic variants}

We downloaded genetic variants from two well-annotated databases (i.e., ClinVar (ver 2020-01-13) [32] and Infevers $[8,34])$. ClinVar is an up-to-date database composed of genetic variants in multiple gens associated with multiple disorders classified as pathogenic, likely pathogenic, benign, likely benign, variant of uncertain significance (VUS), or of conflicting evidence from different sources. We filtered variants that were pathogenic, likely pathogenic, VUS, and conflicting evidence as per ClinVar and retrieved variants in 56 genes associated with 47 autoinflammatory disorders. The Infevers is a publicly available database that [35] has a total of 2112 genetic variants in 38 genes involved in 34 autoinflammatory disorders. The filtered genetic variants from ClinVar and Infever were merged and mapped to the IndiGen filtered variants.

\section{Classification of genetic variants according to ACMG and AMP guidelines}

The American College of Medical Genetics and Genomics and the Association of Molecular Pathology (ACMG-AMP) experts have provided comprehensive guidelines which include annotation of 28 features for variant classification into five broad categories (i.e., pathogenic, likely pathogenic, benign, likely benign, and VUS) [36]. The variant classification was calculated based on the weighted 28 attributes. For variants, pathogenicity was weighted as very strong (PVS1), strong (PS1-4), moderate (PM1-6), or supporting (PP15). Similarly, for benign, it was weighted as stand-alone (BA1), strong (BS1-4), or supporting (BP1-7). The combination of these attributes put together in the algorithm of the genetic variant interpretation tool [37] classifies the variant. The guidelines associated with the weighted attributes have been detailed in Supplementary Data 1.

\section{Statistical significance of pathogenic variants with global population}

The statistical differences in the allele frequencies of pathogenic or likely pathogenic variants were estimated between the Indian population of IndiGen dataset with the global population dataset. The global population datasets include Genome Aggregation Database (gnomAD v3), which is the largest database of 71,702 wholegenome-sequenced individuals from eight populations (African, Amish, Ashkenazi Jews, East Asian, Finnish, Non-Finnish, Latino, and South Asian) [28]; the 1000 Genome Project (1000g2015aug_all) was composed of whole genome sequencing of 2504 individuals from five super populations (Africa, America, Europe, East Asia, and South Asia) [29], and ESP6500 (esp6500siv2_all) composed of 6503 whole exome-sequenced dataset of 2203 African-American and 4300 European-American healthy individuals [38]. The statistical significance was tested by Fisher's exact test; a $P$ value of less than 0.05 was considered significant.

\section{Ancestry haplotyping of a founder mutation}

Since several autoinflammatory conditions have geographical associations, we were interested in the pathogenic or likely pathogenic variants which were prevalent and considered as a founder variant in the population. We further explored the haplotype similarity pattern of the founder variant of each individual from the IndiGen dataset to the global population dataset of the 1000 Genome Project. To evaluate the haplotype similarity, we used a tool called fineSTRUCTURE [39] (version 2.1.3) that uses linkage disequilibrium model-based STRU CTURE approach and principal component analysis for population haplotype prediction. Each individual specific chromosomal variant, where founder variants are located, were merged with 2548 individual variants from the 1000 Genome Project. Further, merged variants were pruned by applying a filter of allele frequency of $>1 \%$ and allele count $>1000$ to get maximum genotype rate using a bespoke bash script. We phased the filtered merged VCF using SHAPEIT v2.r900 tool [40]. Further, the fineSTRU CTURE pipeline was used to analyze the phased VCF and plotted using $\mathrm{R}$ script. The overall workflow adopted in this study has been represented in Fig. 1. 
Table 1 Genes associated with autoinflammatory disorder with their respective mode of inheritance

\begin{tabular}{|c|c|c|}
\hline Gene & Disease & $\begin{array}{l}\text { Mode of } \\
\text { inheritance }\end{array}$ \\
\hline ACP5 & Spondyloenchondrodysplasia with immune dysregulation & AR \\
\hline$A D A 2$ & Deficiency of adenosine deaminase 2 (DADA2) & AR \\
\hline ADAM17 & Inflammatory skin and bowel disease, neonatal & AR \\
\hline ADAR1; ADAR & Aicardi-Goutières syndrome & AR \\
\hline ALPI & Monogenic inflammatory bowel disease & $A R$ \\
\hline AP1S3 & Psoriasis 15 , pustular & $A D$ \\
\hline CARD14 & Familial psoriasis/ CARD14-mediated pustular psoriasis (CAMPS/PSORS2) & $A D$ \\
\hline CDC42 & Takenouchi-Kosaki syndrome & $A D$ \\
\hline COPA & Autoimmune interstitial lung, joint, and kidney disease & $A D$ \\
\hline DNASE1L3 & Systemic lupus erythematosus & AR \\
\hline DNASE2 & Type I interferon-mediated autoinflammation & $A D$ \\
\hline HAVCR2 & T cell lymphoma, subcutaneous panniculitis & $A R$ \\
\hline$|F| H 1$ & Aicardi-Goutières syndrome & $A D$ \\
\hline $1 L 10$ & Interleukin 10 deficiency (IL10D) & $A R$ \\
\hline ILIORA & Inflammatory bowel disease 28 (IBD28)/ interleukin 10 receptor A deficiency (IL10R1D) & AR \\
\hline ILIORB & Inflammatory bowel disease 25 (IBD25)/ interleukin 10 receptor B deficiency (IL10R2D) & AR \\
\hline ILIRN & $\begin{array}{l}\text { Deficiency of interleukin-1ß (IL-1ß) receptor antagonist/ osteomyelitis, sterile multifocal with periostitis pustulosis (DIRA/ } \\
\text { OMPP) }\end{array}$ & AR \\
\hline IL36RN & Deficiency of interleukin-36-receptor antagonist/ generalized pustular psoriasis (GPP)/ (DITRA/PSORP) & AR \\
\hline LACC1 & Juvenile arthritis & AR \\
\hline LPIN2 & MAJEED/ chronic recurrent multifocal osteomyelitis, congenital dyserythropoietic anemia, \& neutrophilic dermatosis & AR \\
\hline MEFV & Familial Mediterranean fever & $A R ; A D$ \\
\hline MVK & Hyperimmunoglobulinemia D with periodic fever syndrome (HIDS); melvanoic aciduria (MA) & AR \\
\hline NCSTN & Acne inversa, familial & $A D$ \\
\hline NLRC4 & Autoinflammation with infantile enterocolitis (AIFEC) & $A D$ \\
\hline NLRP12 & $\begin{array}{l}\text { NLRP12-associated periodic fever syndrome/ familial cold autoinflammatory syndrome 2, or Guadaloupe periodic fever } \\
\text { (NLRP12/FCAS2) }\end{array}$ & $A D$ \\
\hline NLRP1 & Autoinflammation with arthritis and dyskeratosis & $A R ; A D$ \\
\hline NLRP3 & $\begin{array}{l}\text { Familial cold autoinflammatory syndrome (FCAS); Muckle-Wells syndrome (MWS); neonatal onset multisystemic inflamma- } \\
\text { tory disorder/ chronic infantile neurological cutaneous articular syndrome (NOMI/CINCA) }\end{array}$ & $\mathrm{AD}$ \\
\hline NLRP7 & Hydatidiform mole, recurrent, 1 (HYDM1) & $A R$ \\
\hline NOD2 & $\begin{array}{l}\text { Juvenile systemic granulomatosis-Blau syndrome, pediatric granulomatous arthritis (PGA), Crohn's disease early onset } \\
\text { sarcoidosis, or Jabs syndrome (BLAU/PGA/EOS) }\end{array}$ & $A D$ \\
\hline OAS1 & Pulmonary alveolar proteinosis with hypogammaglobulinemia & AR \\
\hline OTULIN & Otulipenia & AR \\
\hline PLCG2 & $\begin{array}{l}\text { PLCG2-associated antibody deficiency and immune dysregulation/ familial atypical cold urticaria (FACU) (PLAID/FCAS3); } \\
\text { autoinflammation and PLCG2-associated antibody deficiency and immune dysregulation (APLAID) }\end{array}$ & $A D$ \\
\hline POLA1 & Type I interferon-mediated autoinflammation & $X L R$ \\
\hline POMP & \multirow{4}{*}{$\begin{array}{l}\text { Chronic atypical neutrophilic dermatosis with lipodystrophy and elevated temperature—Nakajo Nishimura syndrome } \\
\text { (CANDLE/PRAAS) }\end{array}$} & \multirow[t]{4}{*}{ AR } \\
\hline PSMA3 & & \\
\hline $\begin{array}{l}\text { PSMB4 } \\
\text { PSMRB }\end{array}$ & & \\
\hline $\begin{array}{l}\text { PSMB8 } \\
\text { PSMB9 }\end{array}$ & & \\
\hline PSMG2 & Chronic atypical neutrophilic dermatosis with lipodystrophy and elevated temperature (CANDLE) & $A R ; A D$ \\
\hline PSTPIP1 & Pyogenic sterile arthritis, pyoderma gangrenosum and acne syndrome (PAPA) & $\mathrm{AD}$ \\
\hline RBCK1 & Polyglucosan body myopathy, early-onset, with or without immunodeficiency (PBMEI) & AR \\
\hline RNASEH2A & \multirow[t]{3}{*}{ Aicardi-Goutières syndrome } & \multirow[t]{3}{*}{ AR } \\
\hline RNASEH $2 B$ & & \\
\hline RNASEH2C & & \\
\hline SAMHD1 & Chilblain lupus & $A D$ \\
\hline
\end{tabular}


Table 1 (continued)

\begin{tabular}{llc}
\hline Gene & Disease & $\begin{array}{c}\text { Mode of } \\
\text { inheritance }\end{array}$ \\
\hline SH3BP2 & Cherubism & AD \\
SLC29A3 & SLC29A3 spectrum disorder (SLC29A3) & AR \\
TMEM173 & Sting-associated vasculopathy, infantile-onset (SAVI) & AD \\
TNFAIP3 & Autoinflammatory syndrome, familial, Behcet-like (AISBL) & AD \\
TNFRSF11A & TNFRSF11A-associated hereditary fever disease (TRAPS11) & AD \\
TNFRSF1A & TNF receptor-associated periodic syndrome (TRAPS) & AD \\
TREX1 & Systemic lupus erythematosus; Aicardi-Goutières syndrome; Chilblain lupus & AR; AD \\
TRIM22 & Inflammatory bowel disease & AR \\
TRNT1 & Sideroblastic anemia with B cell immunodeficiency, periodic fevers, and developmental delay & AR \\
USP18 & Pseudo-TORCH syndrome & $A R$ \\
WDR1 & Autoinflammatory periodic fever, immunodeficiency, and thrombocytopenia (PFIT) & AR \\
\hline AR autosomat & \\
\hline
\end{tabular}

$A R$ autosomal recessive, $A D$ autosomal dominant, $X L R$ X-linked recessive

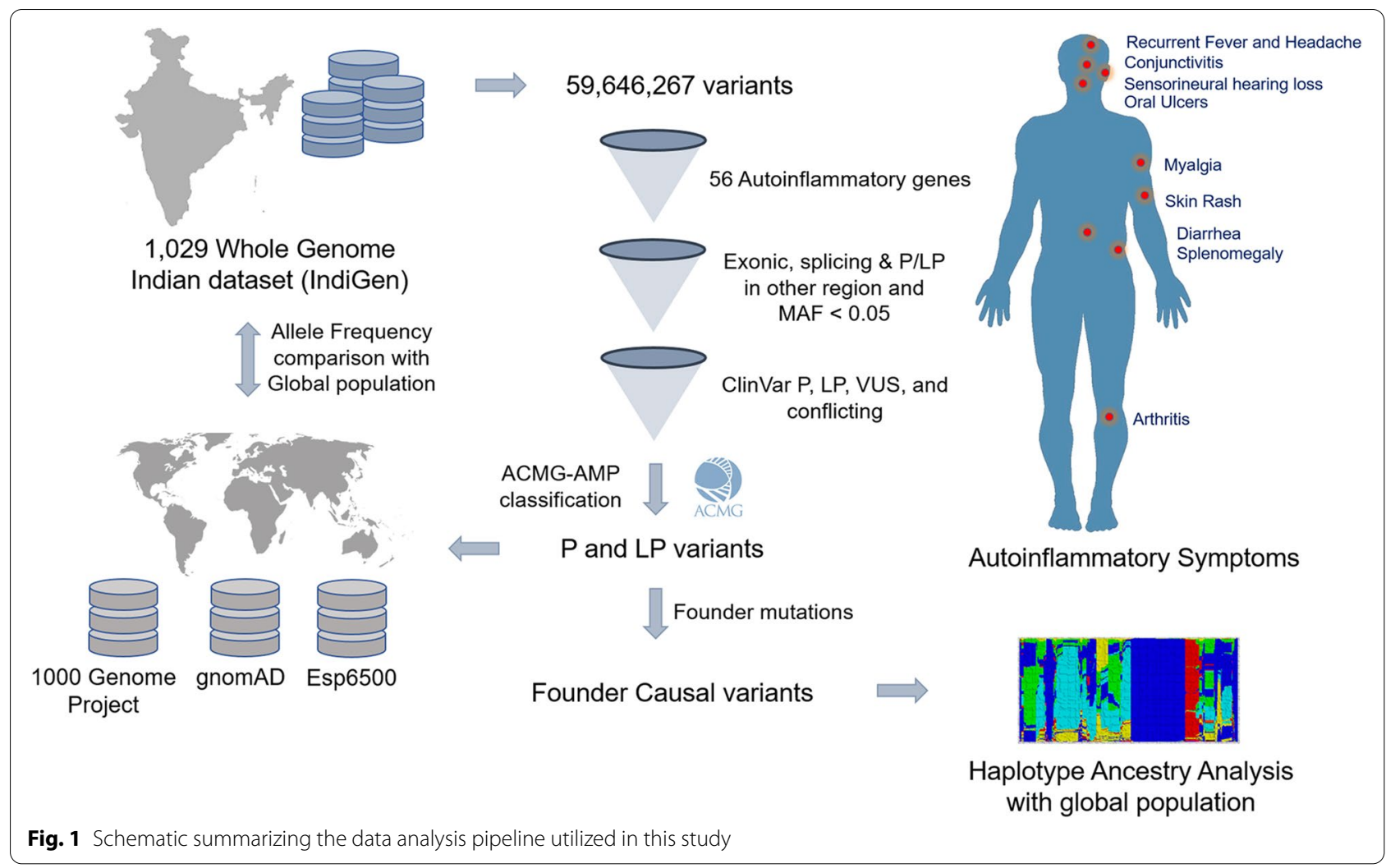

\section{Results}

\section{Genetic variants in the autoinflammatory genes} from different datasets

The IndiGen dataset consisted of a total of 50,517,048 single-nucleotide variants and 5,381,074 InDels, out of which, 110,457 variants were retrieved from 56 genes associated with the 47 autoinflammatory disorders. We further prioritized 871 exonic, splicing and ClinVar pathogenic and likely variants in other regions whose minor allele frequency is less than 0.05 . Further, these variants were mapped on the merged ClinVar pathogenic, likely pathogenic, VUS, and conflicting variants and on Infevers variants that retrieved 166 variants for further analysis as shown in Fig. 2A. 


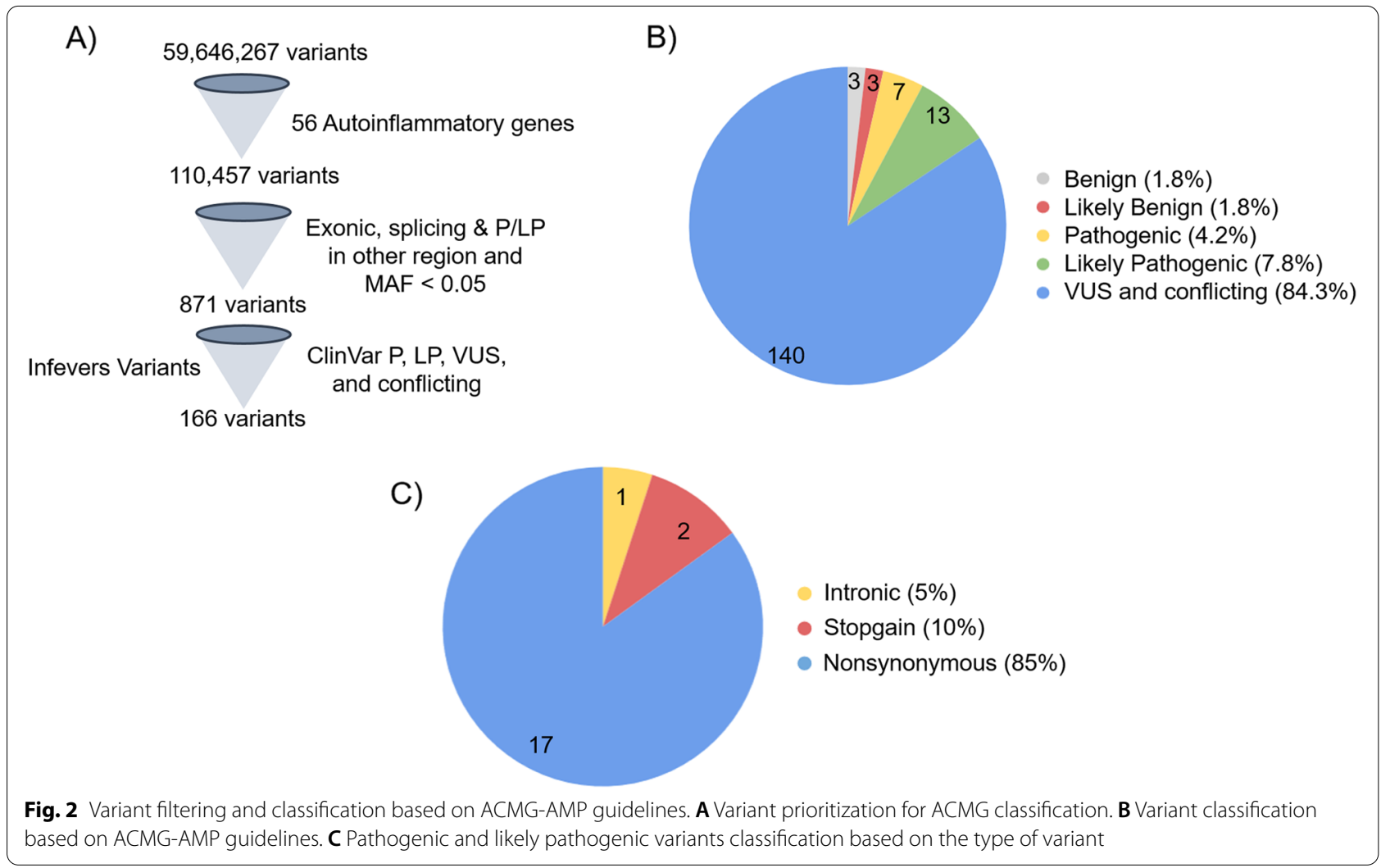

\section{Variant classification based on ACMG and AMP guidelines}

These 166 variants were further scrutinized according to the ACMG-AMP guidelines and classified as pathogenic, likely pathogenic, benign, likely benign, and VUS. In our analysis, we found 7 variants as pathogenic and 13 variants as likely pathogenic (Table 2), while 6 variants were benign and likely benign, and 140 variants were VUS or variants with conflicting evidence (Fig. 2B). Out of 20 pathogenic and likely pathogenic variants, there are 1 intronic, 2 stopgain, and 17 nonsynonymous variants (Fig. 2C). The detailed variant annotation and classification are tabulated in Supplementary Table 1.

\section{Comparison of variant frequency with the global population}

Allele frequencies of 20 pathogenic or likely pathogenic variants were compared with the global populations that include gnomAD V3, 1000 Genome Project (1000g2015aug_all), and ESP6500 (Esp6500siv2_all). We found 17 out of 20 variants were significantly different from the Indian population compared with the global populations (Fisher's exact $P<0.05$ ). All the 17 variants were significantly different in comparison with the gno$\mathrm{mAD}$ database or its subpopulations, while 3 variants had significantly different allele frequencies compared with the 1000 Genomes dataset and 1 variant compared with the Esp6500 European exome dataset. We could not perform Fisher's exact test for 12 variants of the 1000 Genome Project and Esp6500 each as the allele frequencies were unavailable in the respective datasets.

In this analysis, two pathogenic variants rs144478519 and rs148755083 in the IL36RN gene each had $0.1 \%$ allele frequency in the Indian population (IndiGen) that were significantly less in comparison with the European population and East Asian population respectively in both 1000 Genome Project and gnomAD. Interestingly, the latter variant, rs148755083, is private to the East Asian population. Similarly, a pathogenic variant rs78635798 in the RNASEH2C gene had $0.05 \%$ allele frequency in the IndiGen dataset and was present only in the South Asian population. A likely pathogenic variant rs104895492 in the NOD2 gene with high allele frequency in Indian and South Asian population dataset of 0.15 and $0.16 \%$, while absent in other populations. Another likely pathogenic variant rs116107386 in the AP1S3 gene whose allele frequency is significantly low in the Indian population (i.e., $0.05 \%$ in comparison with the four populations of the gnomAD that includes Amish, European (Finnish and Non-Finnish), and Latino as well as European population 
Table 2 Pathogenic and likely pathogenic variants classified by ACMG-AMP guidelines

\begin{tabular}{|c|c|c|c|c|c|c|c|}
\hline Gene & Variant & SNPID & Protein change & ACMG criteria & Classification & Inheritance & Disease \\
\hline ADAR & chr1:154602065:G>C & rs145588689 & c.C577G: p.P193A & PM1, PP3, PM3, PP4 & Likely pathogenic 5 & AR & AGS \\
\hline IL36RN & chr2:113060943:T:C & rs148755083 & $\begin{array}{l}\text { c.115+6T>C: } \\
\text { p.Arg10Argfs X1 }\end{array}$ & PS2, PS3, PP5 & Pathogenic 2 & AR & DITRA \\
\hline IL36RN & chr2:113062547:C>T & rs 144478519 & c.C338T: p.S113L & $\begin{array}{l}\text { PM1, PP3, PS3, PM3, } \\
\text { PP1, PP4 }\end{array}$ & Pathogenic 3b & $A R$ & DITRA \\
\hline AP1S3 & chr2:223777862:A>C & rs116107386 & c.T11G: p.F4C & PM1, PP3, PS3, BS4 & Likely pathogenic 2 & $A D$ & Pustular psoriasis \\
\hline RNASEH2C & chr11:65720385:G>A & rs78635798 & c.C205T:p.R69W & $\begin{array}{l}\text { PM1, PM2, PP3, PP5, } \\
\text { PS3, PP1-M }\end{array}$ & Pathogenic 3a & $A R$ & AGS \\
\hline MVK & chr12:109586107:A>G & rs104895364 & c.A613G: p.N205D & PM1, PM2, PP3, PP4 & Likely pathogenic 5 & $A R$ & HIDS \\
\hline MVK & chr12:109596515:G>A & rs28934897 & c.G1129A: p.V377| & $\begin{array}{l}\text { BP4, PS4, PM3, } \\
\text { PP1-M }\end{array}$ & Likely pathogenic 2 & $A R$ & HIDS \\
\hline RNASEH2B & chr13:50945445:G>A & rs75184679 & c.G529A: p.A177T & $\begin{array}{l}\text { PM1, PP3, PP5, PS3, } \\
\text { BS3, PP4 }\end{array}$ & Likely pathogenic 2 & $A R$ & AGS \\
\hline PSTPIP1 & chr15:77031192:C:T & rs751668240 & c.C850T: p.Q284X & PVS1, PM2, PP3 & Pathogneic 1c & $A D$ & PAPA \\
\hline NOD2 & chr16:50711206:C>T & rs2076754 & c.C1295T: p.A432V & $\begin{array}{l}\text { PM1, PM2, PP3, } \\
\text { BS3, PS4 }\end{array}$ & Likely pathogenic 2 & $A D$ & Blau Syndrome \\
\hline NOD2 & chr16:50711301:G>T & rs104895492 & c.G1390T: p.G464W & PM2, PP3, PS3, PP1 & Likely pathogenic 2 & $A D$ & Blau Syndrome \\
\hline NOD2 & chr16:50712048:C>T & rs104895440 & c.C2137T: p.R713C & PM2, PP3, PS3 & Likely pathogenic 2 & $A D$ & Blau Syndrome \\
\hline CARD14 & chr17:80184015:G>A & rs200731780 & c.G452A: p.R151Q & PM2, BP4, PS3 & Likely pathogenic 2 & $A D$ & CAMPS \\
\hline CARD14 & chr17:80198685:C:T & rs200379060 & c.C1234T: p.R412X & PVS1, PM2 & Likely pathogenic 1 & $A D$ & CAMPS \\
\hline NLRP7 & chr19:54936400:G>A & rs104895525 & c.C2161T: p.R721W & PM2, PP3, PS1 & Likely pathogenic 2 & $A R$ & HYDM1 \\
\hline NLRP12 & chr19:53810605:G:A & rs199881207 & c.C1054T: p.R352C & PM1, PM2, PS3, PP3 & Likely pathogenic 2 & $A D$ & FACS \\
\hline ADA2 & chr22:17203564:G>A & rs148936893 & c.C626T: p.P209L & $\begin{array}{l}\text { PM1, PM2, BP4, PP5, } \\
\text { PS3, PM3, PP1 }\end{array}$ & Pathogenic 3a & $A R$ & DADA2 \\
\hline ADA2 & chr22:17209538:C>A & rs200930463 & c.G140T: p.G47V & $\begin{array}{l}\text { PM1, PM2, PP3, PP5, } \\
\text { PM5 }\end{array}$ & Likely pathogenic 4 & $A R$ & DADA2 \\
\hline ADA2 & chr22:17209539:C>G & rs202134424-G & c.G139C: p.G47R & $\begin{array}{l}\text { PM1, PM2, PP3, PS1, } \\
\text { PS3, PS4 }\end{array}$ & Pathogenic 2 & $A R$ & DADA2 \\
\hline ADA2 & chr22:17209539:C>T & rs202134424-T & c.G139A: p.G47R & $\begin{array}{l}\text { PM1, PM2, PP3, PP5, } \\
\text { PS3, PS4 }\end{array}$ & Pathogenic 2 & $A R$ & DADA2 \\
\hline
\end{tabular}

AGS Aicardi-Goutières syndrome, DITRA deficiency of interleukin-36-receptor antagonist, HIDS hyper IgD syndrome, CAMPS CARD14-mediated pustular psoriasis, HYDM1 hydatidiform mole, recurrent, 1, DADA2 deficiency of adenosine deaminase $2, A R$ autosomal recessive, $A D$ autosomal dominant

of the 1000 Genome Project and ESP6500). An interesting likely pathogenic variant rs28934897 in the $M V K$ gene also popularly known as Dutch mutation has high allele frequency in the Indian population (i.e., 0.34\%, while absent in the South Asian populations of the control population database). The allele frequency, allele count, and allele number of all the pathogenic variants with a comparison with the gnomAD database and its population have been summarized in Table 3. The comparison with all three global populations 1000 Genome Project, gnomAD, and ESP6500 and also with Greater Middle East and Qatar populations 27,408,750 has been tabulated in Supplementary Table 2 and well represented in Fig. 3.

\section{Ancestry haplotyping}

We found six causal founder variants (i.e., rs28934897, rs148755083, rs202134424-T, rs202134424-G, rs200930463, and rs78635798 in the $M V K, I L 36 R N, A D A 2, A D A 2, A D A 2$, and $R N A S E H 2 C$ genes, respectively). The first three variants rs28934897, rs202134424-T, and rs148755083 were present in seven, four, and two individuals, respectively while other three variants (rs202134424-G, rs200930463, and rs78635798) were found in a single individual of the IndiGen dataset in heterozygous state. To identify the haplotype ancestry, we have applied filters of allele frequency $>1 \%$ on the merged IndiGen individual variant with the 1000 Genome Project that results on an average of 23,843, 784,354, 137,671, 137,701, 137,783, and 473,514 variants with approximately $99 \%$ genotype rate for variants rs28934897, rs148755083, rs202134424-T, rs202134424-G, rs200930463, and rs78635798, respectively. After performing chromosomal painting using fineSTRUCTURE, we identified six out of seven individuals had admixed European haplotype around the rs28934897 variant along with South Asian in four individuals and American haplotype in 
Table 3 IndiGen allele frequency comparison $P$ value $(<0.05)$ of autoinflammatory disorder pathogenic and likely pathogenic variants with the gnomAD (global) database

\begin{tabular}{|c|c|c|c|c|c|c|c|c|c|c|c|c|}
\hline \multirow[b]{2}{*}{$\begin{array}{c}\text { Pathogenic } \\
\text { variant }\end{array}$} & \multirow[b]{2}{*}{ Allele } & \multirow[b]{2}{*}{ IndiGen } & \multirow[b]{2}{*}{$\begin{array}{c}\text { gnomA } \\
\text { D All }\end{array}$} & \multicolumn{9}{|c|}{ gnom AD } \\
\hline & & & & AMI & $\begin{array}{l}\text { EUR } \\
\text { (Fin) }\end{array}$ & $\begin{array}{c}\text { EUR } \\
\text { (Non- } \\
\text { Fin) }\end{array}$ & Latino & AFR & ASJ & EAS & SAS & Other \\
\hline chr1-154602065 & Ref: G & 0.9980 & 0.998 & 1.000 & 1.000 & 0.997 & 0.996 & 0.999 & 0.999 & 1.000 & 0.999 & 0.997 \\
\hline rs145588689 & Alt: C & 0.0020 & 0.002 & 0.000 & 0.001 & 0.004 & 0.004 & 0.001 & 0.001 & 0.000 & 0.001 & 0.003 \\
\hline \multirow[t]{2}{*}{ ADAR } & $\mathrm{AC} / \mathrm{AN}$ & $4 / 2050$ & $327 / 143292$ & $0 / 900$ & $5 / 10484$ & 224 / 64564 & $54 / 13656$ & 34 / 42038 & $2 / 3324$ & $0 / 3134$ & $2 / 3042$ & $6 / 2150$ \\
\hline & P-value: & & 1.000 & 0.321 & $0.045^{*}$ & 0.334 & 0.238 & 0.099 & 0.211 & $0.02^{*}$ & 0.228 & 0.754 \\
\hline chr2-113062547 & Ref: C & 0.9990 & 0.997 & 1.000 & 0.994 & 0.996 & 0.997 & 0.999 & 1.000 & 1.000 & 0.999 & 0.997 \\
\hline rs144478519 & Alt: $\mathrm{T}$ & 0.0010 & 0.003 & 0.000 & 0.006 & 0.004 & 0.003 & 0.001 & 0.000 & 0.000 & 0.001 & 0.003 \\
\hline \multirow[t]{2}{*}{ IL36RN } & $\mathrm{AC} / \mathrm{AN}$ & $2 / 2048$ & 372 / 143266 & $0 / 900$ & $64 / 10476$ & $233 / 64558$ & $42 / 13660$ & $25 / 42026$ & $0 / 3320$ & $0 / 3134$ & $2 / 3044$ & $6 / 2148$ \\
\hline & P-value: & & 0.187 & 1.000 & $0.001^{*}$ & 0.054 & 0.115 & 0.359 & 0.146 & 0.156 & 1.000 & 0.290 \\
\hline chr2-113060943 & Ref: $T$ & 0.999 & 1.000 & 1.000 & 1.000 & 1.000 & 1.000 & 1.000 & 1.000 & 0.991 & 1.000 & 1.000 \\
\hline rs148755083 & Alt: C & 0.001 & 0.000 & 0.000 & 0.000 & 0.000 & 0.000 & 0.000 & 0.000 & 0.009 & 0.000 & 0.000 \\
\hline \multirow[t]{2}{*}{ IL36RN } & $\mathrm{AC} / \mathrm{AN}$ & $2 / 2052$ & 29/143252 & $0 / 900$ & $0 / 10464$ & $0 / 64562$ & $0 / 13656$ & 0/ 3324 & 0/ 3324 & $29 / 3130$ & 0/ 3046 & 0/ 2152 \\
\hline & P-value: & & 0.071 & 1.000 & $0.026^{*}$ & $0.001^{*}$ & $0.017^{\star}$ & 0.146 & 0.146 & $0.000055^{\star}$ & 0.162 & 0.238 \\
\hline chr2-223777862 & Ref: A & 0.99951 & 0.991 & 0.978 & 0.993 & 0.986 & 0.993 & 0.997 & 0.996 & 1.000 & 0.999 & 0.986 \\
\hline rs116107386 & Alt: C & 0.0005 & 0.009 & 0.022 & 0.007 & 0.014 & 0.007 & 0.003 & 0.004 & 0.000 & 0.001 & 0.014 \\
\hline \multirow[t]{2}{*}{ AP1S3 } & $\mathrm{AC} / \mathrm{AN}$ & $1 / 2048$ & 1264 / 143304 & $20 / 900$ & 907 / 64574 & $71 / 10482$ & $94 / 13666$ & $125 / 42030$ & $13 / 3322$ & $0 / 3132$ & $4 / 3048$ & $30 / 2150$ \\
\hline & P-value: & & $7.52 \mathrm{E}-07^{\star}$ & $8.59 \mathrm{E}-10^{\star}$ & 3.33E-11* & $0.00007^{\star}$ & $0.00004^{*}$ & 0.032 & 0.023 & 0.396 & 0.654 & 3.84E-08* \\
\hline chr11-65720385 & Ref: G & 0.99951 & 1.000 & 1.000 & 1.000 & 1.000 & 1.000 & 1.000 & 1.000 & 1.000 & 0.999 & 1.000 \\
\hline rs78635798 & Alt: A & 0.0005 & 0.000 & 0.000 & 0.000 & 0.000 & 0.000 & 0.000 & 0.000 & 0.000 & 0.001 & 0.000 \\
\hline \multirow[t]{2}{*}{ RNASEH2C } & $\mathrm{AC} / \mathrm{AN}$ & $1 / 2038$ & $2 / 143340$ & $0 / 898$ & $0 / 10482$ & $0 / 64574$ & $0 / 13666$ & $0 / 42068$ & $0 / 3322$ & $0 / 3130$ & $2 / 3048$ & $0 / 2152$ \\
\hline & P-value: & & $0.0415^{\star}$ & 1.000 & 0.163 & $0.03^{*}$ & 0.130 & $0.046^{*}$ & 0.380 & 0.395 & 1.000 & 0.487 \\
\hline chr12-109586107 & Ref: A & 0.99951 & 1.000 & 1.000 & 1.000 & 1.000 & 1.000 & 1.000 & 1.000 & 1.000 & 1.000 & 1.000 \\
\hline rs104895364 & Alt: $\mathrm{G}$ & 0.0005 & 0.000 & 0.000 & 0.000 & 0.000 & 0.000 & 0.000 & 0.000 & 0.000 & 0.000 & 0.000 \\
\hline \multirow[t]{2}{*}{ MVK } & $\mathrm{AC} / \mathrm{AN}$ & $1 / 2048$ & $2 / 143326$ & $0 / 900$ & $0 / 10478$ & $0 / 64574$ & $1 / 13664$ & $1 / 42054$ & $0 / 3322$ & $0 / 3130$ & $0 / 3050$ & $0 / 2154$ \\
\hline & P-value: & & $0.04169^{*}$ & 1.000 & 0.164 & $0.031^{*}$ & 0.244 & 0.091 & 0.382 & 0.396 & 0.402 & 0.488 \\
\hline chr12-109596515 & Ref: G & 0.99658 & 0.999 & 1.000 & 0.999 & 0.998 & 0.999 & 1.000 & 0.999 & 1.000 & 1.000 & 0.999 \\
\hline rs28934897 & Alt: A & 0.0034 & 0.002 & 0.000 & 0.001 & 0.003 & 0.001 & 0.000 & 0.001 & 0.000 & 0.000 & 0.001 \\
\hline \multirow[t]{2}{*}{ MVK } & $\mathrm{AC} / \mathrm{AN}$ & $7 / 2046$ & $219 / 143294$ & $0 / 898$ & $15 / 10474$ & 164 / 64572 & $18 / 13654$ & 17 / 42046 & $3 / 3322$ & $0 / 3132$ & $0 / 3044$ & $2 / 2152$ \\
\hline & P-value: & & $0.0425^{\star}$ & 0.109 & 0.075 & 0.375 & $0.037^{\star}$ & $0.00008^{\star}$ & 0.050 & $0.002^{*}$ & $0.002^{*}$ & 0.101 \\
\hline chr13-50945445 & Ref: G & 0.99951 & 0.999 & 1.000 & 1.000 & 0.998 & 0.997 & 1.000 & 1.000 & 1.000 & 0.999 & 0.998 \\
\hline rs75184679 & Alt: A & 0.0005 & 0.001 & 0.000 & 0.000 & 0.002 & 0.003 & 0.000 & 0.000 & 0.000 & 0.001 & 0.002 \\
\hline \multirow[t]{2}{*}{ RNASEH2B } & $\mathrm{AC} / \mathrm{AN}$ & $1 / 2046$ & $200 / 143276$ & $0 / 900$ & $2 / 10478$ & $141 / 64562$ & $38 / 13654$ & 12 / 42036 & $1 / 3324$ & $0 / 3134$ & $2 / 3034$ & $4 / 2154$ \\
\hline & P-value: & & 0.538 & 1.000 & 0.415 & 0.138 & 0.054 & 0.461 & 1.000 & 0.395 & 1.000 & 0.376 \\
\hline chr15-77031192 & Ref: C & 0.99951 & 1.000 & 1.000 & 1.000 & 1.000 & 1.000 & 1.000 & 1.000 & 1.000 & 1.000 & 1.000 \\
\hline rs751668240 & Alt: $T$ & 0.0005 & 0.000 & 0.000 & 0.000 & 0.000 & 0.000 & 0.000 & 0.000 & 0.000 & 0.000 & 0.000 \\
\hline \multirow[t]{2}{*}{ PSTPIP1 } & $\mathrm{AC} / \mathrm{AN}$ & $1 / 2046$ & $4 / 143332$ & $0 / 898$ & $0 / 10478$ & $1 / 64578$ & $3 / 13664$ & $0 / 42060$ & $0 / 3318$ & $0 / 3134$ & $0 / 3050$ & $0 / 2152$ \\
\hline & P-value: & & 0.068 & 1.000 & 0.163 & 0.061 & 0.428 & $0.046^{\star}$ & 0.382 & 0.395 & 0.402 & 0.487 \\
\hline chr16-50711206 & Ref: C & 0.99951 & 1.000 & 1.000 & 1.000 & 1.000 & 1.000 & 1.000 & 1.000 & 1.000 & 1.000 & 1.000 \\
\hline rs2076754 & Alt: $T$ & 0.0005 & 0.000 & 0.000 & 0.000 & 0.001 & 0.000 & 0.000 & 0.000 & 0.000 & 0.000 & 0.001 \\
\hline \multirow[t]{2}{*}{ NOD2 } & $\mathrm{AC} / \mathrm{AN}$ & $1 / 2048$ & $33 / 143338$ & $0 / 900$ & $0 / 10480$ & 32 / 64582 & $0 / 13666$ & $0 / 42050$ & $0 / 3324$ & $0 / 3134$ & $0 / 3048$ & $1 / 2154$ \\
\hline & P-value: & & 0.383 & 1.000 & 0.164 & 1.000 & 0.130 & $0.046^{*}$ & 0.381 & 0.395 & 0.402 & 1.000 \\
\hline chr16-50711301 & Ref: G & 0.99854 & 1.000 & 1.000 & 1.000 & 1.000 & 1.000 & 1.000 & 1.000 & 1.000 & 0.998 & 1.000 \\
\hline rs104895492 & Alt: $T$ & 0.0015 & 0.000 & 0.000 & 0.000 & 0.000 & 0.000 & 0.000 & 0.000 & 0.000 & 0.002 & 0.000 \\
\hline NOD2 & $\mathrm{AC} / \mathrm{AN}$ & $3 / 2048$ & $5 / 143312$ & $0 / 900$ & $0 / 10482$ & $0 / 64570$ & $0 / 13662$ & $0 / 42044$ & $0 / 3324$ & $0 / 3128$ & $5 / 3050$ & $0 / 2152$ \\
\hline
\end{tabular}


Table 3 (continued)

\begin{tabular}{|c|c|c|c|c|c|c|c|c|c|c|c|c|}
\hline \multirow[b]{2}{*}{$\begin{array}{l}\text { Pathogenic } \\
\text { variant }\end{array}$} & \multirow[b]{2}{*}{ Allele } & \multirow[b]{2}{*}{ IndiGen } & \multirow[b]{2}{*}{$\begin{array}{c}\text { gnomA } \\
\text { D All }\end{array}$} & \multicolumn{9}{|c|}{ gnomAD } \\
\hline & & & & AMI & $\begin{array}{l}\text { EUR } \\
\text { (Fin) }\end{array}$ & $\begin{array}{l}\text { (Non- } \\
\text { Fin) }\end{array}$ & Latino & AFR & ASJ & EAS & SAS & Other \\
\hline & P-value: & & $0.00015^{\star}$ & 0.558 & $0.004^{*}$ & $0.00003^{*}$ & $0.00222^{*}$ & $0.0001^{*}$ & 0.056 & 0.062 & 1.000 & 0.116 \\
\hline chr16-50712048 & Ref: C & 0.99951 & 1.000 & 1.000 & 1.000 & 1.000 & 1.000 & 1.000 & 1.000 & 1.000 & 1.000 & 1.000 \\
\hline rs104895440 & Alt: T & 0.0005 & 0.000 & 0.000 & 0.000 & 0.000 & 0.000 & 0.000 & 0.000 & 0.000 & 0.000 & 0.000 \\
\hline \multirow[t]{2}{*}{ NOD2 } & $\mathrm{AC} / \mathrm{AN}$ & $1 / 2044$ & $3 / 143366$ & $0 / 900$ & $0 / 10488$ & $2 / 64592$ & $1 / 13658$ & $0 / 42070$ & $0 / 3322$ & $0 / 3134$ & $0 / 3048$ & $0 / 2154$ \\
\hline & P-value: & & 0.055 & 1.000 & 0.163 & 0.089 & 0.244 & $0.046^{*}$ & 0.381 & 0.395 & 0.402 & 0.487 \\
\hline chr17-80184015 & Ref: $G$ & 0.99951 & 1.000 & 1.000 & 1.000 & 1.000 & 1.000 & 1.000 & 1.000 & 1.000 & 0.999 & 1.000 \\
\hline rs200731780 & Alt: A & 0.0005 & 0.000 & 0.000 & 0.000 & 0.000 & 0.000 & 0.000 & 0.000 & 0.000 & 0.001 & 0.000 \\
\hline \multirow[t]{2}{*}{ CARD14 } & $\mathrm{AC} / \mathrm{AN}$ & $1 / 2046$ & $5 / 143066$ & $0 / 894$ & $1 / 10436$ & $1 / 64494$ & $1 / 13646$ & $0 / 41946$ & $0 / 3324$ & $0 / 3132$ & $2 / 3044$ & $0 / 2150$ \\
\hline & P-value: & & 0.082 & 1.000 & 0.301 & 0.061 & 0.244 & $0.046^{*}$ & 0.381 & 0.395 & 1.000 & 0.488 \\
\hline chr17-80198685 & Ref: C & 0.99951 & 1.000 & 1.000 & 1.000 & 1.000 & 1.000 & 0.999 & 1.000 & 1.000 & 1.000 & 1.000 \\
\hline rs200379060 & Alt: T & 0.0005 & 0.000 & 0.000 & 0.000 & 0.000 & 0.000 & 0.001 & 0.000 & 0.000 & 0.000 & 0.000 \\
\hline \multirow[t]{2}{*}{ CARD14 } & $\mathrm{AC} / \mathrm{AN}$ & $1 / 2046$ & $38 / 143348$ & 0/900 & 0/10484 & $1 / 64582$ & $2 / 13662$ & $33 / 42056$ & $0 / 3324$ & $450,711.000$ & 0/3052 & $92,774.000$ \\
\hline & P-value: & & 0.425 & 1.000 & 0.163 & 0.061 & 0.342 & 1.000 & 0.381 & 1.000 & 0.401 & 1.000 \\
\hline chr19-54936400 & Ref: $G$ & 0.99951 & 1.000 & 1.000 & 1.000 & 1.000 & 1.000 & 1.000 & 1.000 & 1.000 & 1.000 & 1.000 \\
\hline rs 104895525 & Alt: A & 0.0005 & 0.000 & 0.000 & 0.000 & 0.000 & 0.000 & 0.000 & 0.000 & 0.000 & 0.000 & 0.000 \\
\hline \multirow[t]{2}{*}{ NLRP7 } & $\mathrm{AC} / \mathrm{AN}$ & $1 / 2048$ & $3 / 143298$ & $0 / 900$ & $0 / 10474$ & $2 / 64580$ & $0 / 13632$ & $0 / 42058$ & $0 / 3324$ & $0 / 3132$ & $1 / 3046$ & $0 / 2152$ \\
\hline & P-value: & & 0.055 & 1.000 & $0.0044^{*}$ & $0.0000128^{*}$ & $0.00004^{*}$ & $1.01 \mathrm{E}-08^{*}$ & $0.0012^{\star}$ & $0.0006^{*}$ & $0.0018^{*}$ & $0.0008^{*}$ \\
\hline chr19:53810605 & Ref: $G$ & 0.999 & 1.000 & 1.000 & 1.000 & 1.000 & 1.000 & 1.000 & 1.000 & 1.000 & 0.999 & 1.000 \\
\hline rs199881207 & Alt: A & 0.0010 & 0.000 & 0.000 & 0.000 & 0.000 & 0.000 & 0.000 & 0.000 & 0.000 & 0.001 & 0.000 \\
\hline \multirow[t]{2}{*}{ NLRP12 } & $\mathrm{AC} / \mathrm{AN}$ & $2 / 2044$ & $29 / 143174$ & $0 / 898$ & $2 / 10480$ & 22 / 64530 & $1 . / 13626$ & $1 / 42000$ & $0 / 3320$ & $0 / 3130$ & $3 / 3036$ & $0 / 2154$ \\
\hline & P-value: & & 0.070 & 1.000 & 0.127 & 0.167 & $0.0466^{*}$ & $0.006^{\star}$ & 0.145 & 0.156 & 1.000 & 0.237 \\
\hline chr22-17203564 & Ref: $G$ & 0.99951 & 1.000 & 1.000 & 1.000 & 1.000 & 1.000 & 1.000 & 1.000 & 1.000 & 1.000 & 1.000 \\
\hline rs148936893 & Alt: A & 0.0005 & 0.000 & 0.000 & 0.000 & 0.000 & 0.000 & 0.000 & 0.000 & 0.000 & 0.000 & 0.000 \\
\hline \multirow[t]{2}{*}{ ADA2 } & $\mathrm{AC} / \mathrm{AN}$ & $1 / 2050$ & $5 / 143304$ & $0 / 900$ & $0 / 10478$ & $3 / 64576$ & $1 / 13648$ & $1 / 42046$ & $0 / 3324$ & $0 / 3134$ & $0 / 3048$ & $0 / 2150$ \\
\hline & P-value: & & 0.082 & 1.000 & 0.164 & 0.118 & 0.244 & 0.091 & 0.382 & 0.396 & 0.402 & 0.488 \\
\hline chr22-17209538 & Ref: C & 0.99951 & 1.000 & 1.000 & 1.000 & 1.000 & 1.000 & 1.000 & 1.000 & 1.000 & 1.000 & 1.000 \\
\hline rs200930463 & Alt: A & 0.0005 & 0.000 & 0.000 & 0.000 & 0.000 & 0.000 & 0.000 & 0.000 & 0.000 & 0.000 & 0.000 \\
\hline \multirow[t]{2}{*}{ ADA2 } & $\mathrm{AC} / \mathrm{AN}$ & $1 / 2052$ & $1 / 143248$ & $0 / 900$ & $0 / 10458$ & $1 / 64574$ & $0 / 13634$ & 0/42032 & $0 / 3320$ & $0 / 3130$ & $0 / 3048$ & $0 / 2152$ \\
\hline & P-value: & & $0.0281^{*}$ & 1.000 & 0.164 & 0.061 & 0.131 & $0.046^{*}$ & 0.382 & 0.396 & 0.403 & 0.488 \\
\hline chr22-17209539 & Ref: C & 0.99951 & 1.000 & 1.000 & 1.000 & 1.000 & 1.000 & 1.000 & 1.000 & 1.000 & 1.000 & 1.000 \\
\hline rs202134424 & Alt: G & 0.0005 & 0.000 & 0.000 & 0.000 & 0.000 & 0.000 & 0.000 & 0.000 & 0.000 & 0.000 & 0.000 \\
\hline \multirow[t]{2}{*}{ ADA2 } & $\mathrm{AC} / \mathrm{AN}$ & $1 / 2052$ & $0 / 143248$ & $0 / 900$ & $0 / 10458$ & $0 / 64574$ & $0 / 13634$ & 0/42032 & $0 / 3320$ & $0 / 3130$ & $0 / 3048$ & $0 / 2152$ \\
\hline & P-value: & & $0.0141^{*}$ & 1.000 & 0.164 & $0.0308^{*}$ & 0.131 & $0.046^{\star}$ & 0.382 & 0.396 & 0.403 & 0.488 \\
\hline chr22-17209539 & Ref: C & 0.99854 & 1.000 & 1.000 & 1.000 & 1.000 & 1.000 & 1.000 & 1.000 & 1.000 & 0.999 & 1.000 \\
\hline rs202134424 & Alt: A & 0.00146 & 0.000 & 0.000 & 0.000 & 0.000 & 0.000 & 0.000 & 0.000 & 0.000 & 0.001 & 0.000 \\
\hline \multirow[t]{2}{*}{ ADA2 } & $\mathrm{AC} / \mathrm{AN}$ & 3 / 2052 & $6 / 143216$ & $0 / 900$ & $0 / 10458$ & $2 / 64574$ & $1 / 13622$ & $0 / 42026$ & $0 / 3318$ & $0 / 3130$ & $3 / 3038$ & $0 / 2150$ \\
\hline & P-value: & & $0.00022^{*}$ & 0.558 & $0.0044^{\star}$ & $0.00027^{\star}$ & $0.0081^{*}$ & $0.0001^{*}$ & 0.056 & 0.062 & 0.691 & 0.117 \\
\hline
\end{tabular}

AMI Amish, EUR (Fin) European (Finnish), EUR (Non-Fin) European (Non-Finnish), AFR African, ASJ Ashkenazi Jewish, EAS East Asian, SAS South Asian Significant values are marked with asterisk ${ }^{*}$ ) and cells colored in red

two individuals, while one individual had a complete South Asian haplotype. Similarly, those with variant rs202134424G/T had admixed European haplotype in three individuals, out of which two had admixed American and one had admixed South Asian haplotype, while one individual had complete South Asian haplotype. Interestingly, a variant rs148755083 identified in two individuals in IndiGen was found to have East Asian haplotype. Also, the variant rs200930463 harbored by an IndiGen individual was found to have East Asian haplotype. While an individual harboring a variant rs78635798 had a South Asian haplotype around the variant. The painted chromosomal region $500 \mathrm{~KB}$ upstream and downstream of the founder variant has been represented in Fig. 4. 


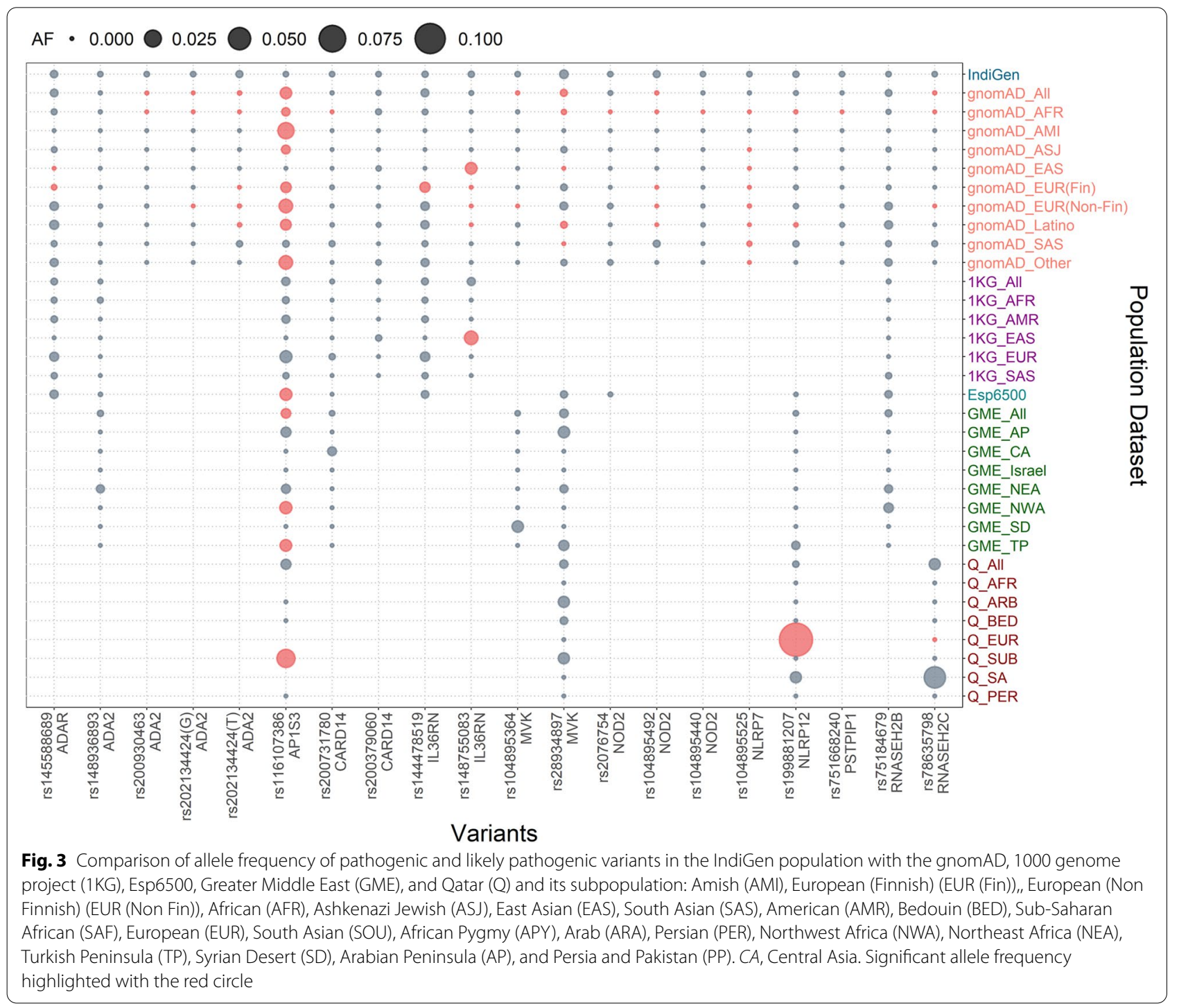

\section{Discussion}

Autoinflammatory disorders are a group of Mendelian disorders caused by genetic defects in the genes involved in the regulation of innate immune systems. There are more than 50 genes that are associated with the autoinflammatory disorders as curated by the experts of the International Union of Immunological Societies (IUIS) [33] and Infevers [8, 34]. There are a number of genetic epidemiological studies that have been carried out around the world which suggest high prevalence of the distinct autoinflammatory disorders in different regions of the world [41-46]. The most common autoinflammatory disorder is familial Mediterranean fever (FMF) found to be very common in Middle Eastern countries [42, 44, 45]. A study performed on 1299 Armenian patients affected with FMF found a high likelihood of carrier individuals with disease manifestation [45]. Another study revealed the carrier with high frequency of $M E F V$ mutation (i.e., 1 in 5 in healthy Armenian individuals) [44]. A comprehensive study performed by our group on more than 2000 whole exome sequence dataset of the Mediterranean region revealed a carrier frequency of $8 \%$ in the population [41]. The 8-year epidemiological retrospective study for the cryopyrin-associated periodic syndrome (CAPS) caused due to the NLRP3 gene estimated 1/360,000 prevalence in France [43]. Another study performed by Houten et al. estimated 1:65 carrier frequency of $M V K$ mutation in the Dutch population associated with hyper IgD syndrome [46]. While only a handful of genetically characterized autoinflammatory diseases have been reported in India [22, 47] including from our group [48], the genetic frequencies of variants 


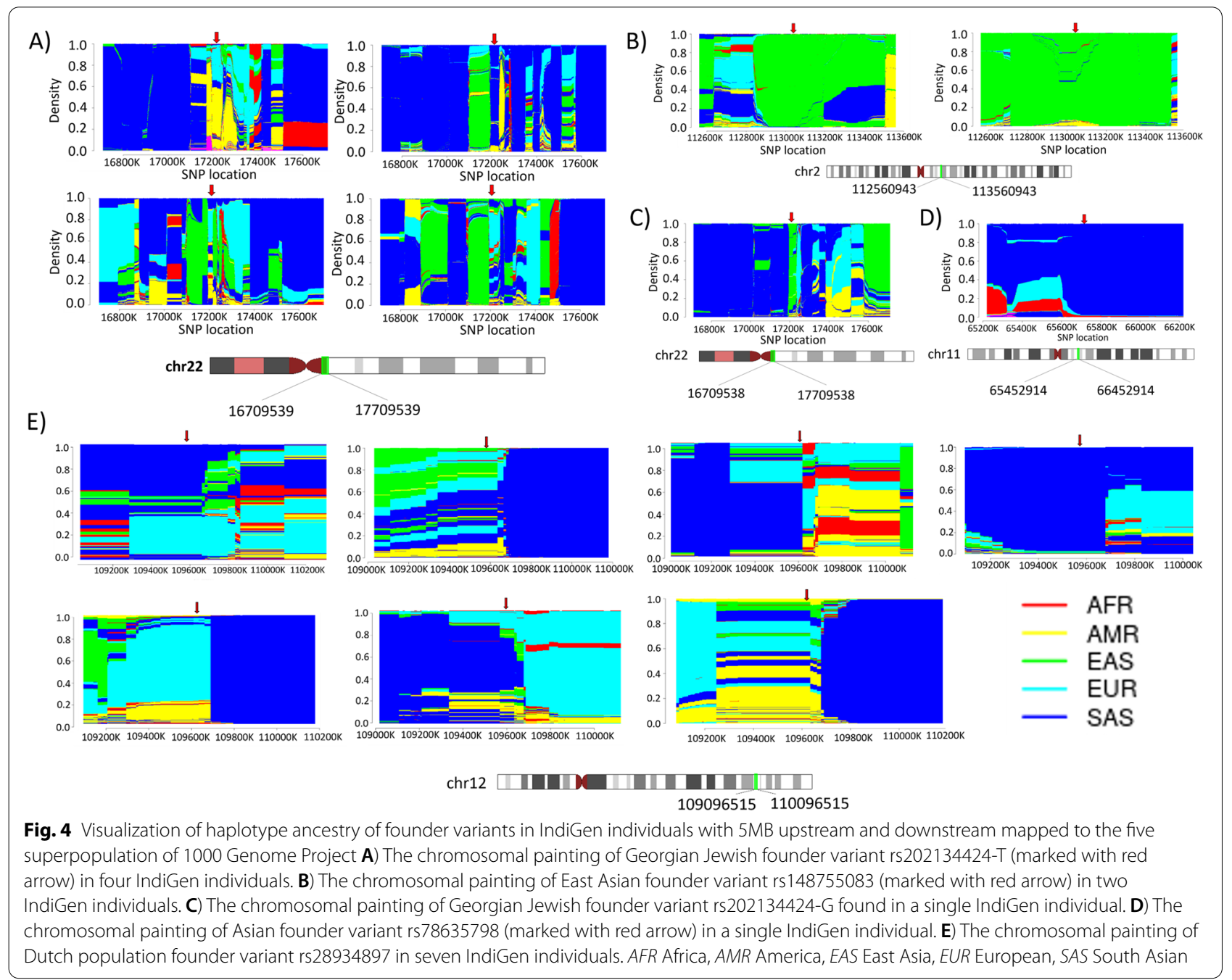

in the population remain an enigma. The recent availability of genome-wide data for the cosmopolitan Indian population has motivated us to understand the genetic epidemiology of autoinflammatory diseases in India. Our systematic analysis of variants and reclassification according to the ACMG \& AMP guidelines revealed a total of 20 genetic variants which could be classified as pathogenic or likely pathogenic.

In total, 36 genomes in the IndiGen dataset had at least one pathogenic/likely pathogenic variant. Of the individuals with carriers for any of the pathogenic/ likely pathogenic variants, in the $A D A 2$ genes, causing deficiency of adenosine deaminase 2 (DADA2) had the maximum number of 4 unique variants in 6 individuals. The second maximum number of variants that was present in the NOD2 gene that causes Blau syndrome and had 3 unique variants in 5 individuals. This was followed by the $M V K$, NLRP12, and IL36RN genes causing hyper IgD syndrome, (HIDS) and generalized pustular psoriasis (GPP) with
2 unique variants each, in 8 and 4 individuals, respectively. A total of 4 and 2 individuals were carriers for a causal variant in the ADAR and NLRP12 gene causative for Aicardi-Goutières syndrome (AGS) and familial cold autoinflammatory syndrome (FACS). While the remaining 7 genes (AP1S3, RNASEH2C, RNASEH2B, CARD14, NLRP7, PSTPIP1, and CARD14) have only a single individual carrier for the causal variant.

Out of 20 causal variants, 7 were pathogenic (1 intronic, 1 stopgain, and 5 nonsynonymous), and 13 are likely pathogenic variants (1 stopgain and 12 nonsynonymous) by ACMG-AMP guidelines. We identified two pathogenic variants in the IL36RN gene that includes a nonsynonymous variant (c.338C $>$ T: p.S113L) rs144478519 and intronic variant rs148755083 (c.115+6T $>$ C: p.Arg10ArgfsX1). A nonsynonymous variant rs144478519 was found in multiple unrelated patients of different ancestries affected with GPP in the homozygous or compound heterozygous state. This 
variant falls in a region that is evolutionarily conserved with proximity to the binding site involved in receptor interaction. This interaction is responsible for the IL-36 signaling system for the inhibition of the activity of interleukin-36 that further inhibits the local autoinflammatory response [23]. Functional studies also revealed a significant increase in proinflammatory cytokines [49-51]. The latter intronic variant rs148755083 is predominantly found in the East Asian populations with allele frequency of $\sim 1 \%$ in the global dataset. This variant has been found to be highly prevalent in patients of Japanese and Chinese ancestry and was identified as founder mutation in both the population [50, 52-54]. A nonsynonymous pathogenic variant rs78635798 (c.205C > T: p.R69W) in the RNASEH2C gene causative of AGS had very low allele frequency in the global populations (i.e., $<0.01 \%$ ) and falls in the functionally important domain. Functional studies revealed $R N A S E H 2 C$ p.R69W had a significant reduction in the thermal stability of RNase H2 complex [55]. This variant has been recurrent and considered as a founder mutation in the Asian population as well; it segregates with the disease in the family [56]. Other founder pathogenic variants from the Georgian Jewish population (p.G47R) rs200930463-T and rs200930463$\mathrm{G}$ in the ADA2 gene cause DADA2 with the carrier frequency in this population as $10.2 \%$ with the high prevalence of the disease [57]. However, in the global population datasets, it has a very low allele frequency of $<0.0002 \%$. The functional analysis showed a marked reduction of ADA2 activity in comparison with the wild type in the homozygous state $[57,58]$. Recently, a case series of 33 DADA2-affected patients from India has been reported and found this variant p.G47R to be prevalent in the Jain/Aggarwal community [59]. Another $A D A 2$ variant at the same amino acid position of the ADA2 protein (p.G47V) rs200930463 was in a trans compound heterozygous state with p.W246S in the patient affected with DADA2 [57]. Also, functional studies revealed the complete absence of ADA2 protein in cells transfected with p.G47V and p.W246S as well as lower amounts in drosophila S2 cells [57]. The pathogenic nonsynonymous variant rs148936893 (c.C752T: p.P251L) in the ADA2 gene associated with the DADA2 has a low frequency of $<0.02 \%$ in the global population datasets. This variant was found to be segregated with the disease in a German family in a trans compound heterozygous state. Functional studies revealed ADA2 activity has been severely compromised and also indicated intracellular elevated levels of ADA2 protein [57].

Another founder mutation of Dutch ancestry (c.G1129A: p.V377I) rs28934897 in the $M V K$ gene causes HIDS with very high carrier frequency (i.e.,
1:65) [46]. This variant has been reported in multiple patients with HIDS of different ancestry and found variant segregation with the disease in the family either in compound heterozygous or homozygous state [60-62]. In vivo and in vitro functional studies have revealed a significant decrease in the enzymatic activity of MVK protein [63]. Recently, we have found a p.V377I variant in our six patients of South India ancestry in a trans compound heterozygous state residing in the same region and identified as founder in the South Indian population [48]. Another likely pathogenic $M V K$ variant rs104895364 (c.613A>G: p.N205D) was found in a trans compound heterozygous state in two patients affected with HIDS of the same family [48]. It has a very low allele frequency of $<0.005 \%$ in the global population datasets (i.e., 1000 Genome Project, gnomAD, and Esp6500). The variant falls in the functionally important domain and was found in multiple patients affected with HIDS of different ancestry [64-67]. A likely pathogenic nonsynonymous variant rs 145588689 (c.577C>G: p.P193A) in the ADAR gene associated with AicardiGoutières syndrome (AGS) was found to be segregated with disease in compound heterozygous state in 22 out of 23 unrelated families as well as in multiple unrelated AGS patients $[68,69]$. This variant falls in the functional domain that disrupts the interaction between Z-DNA/ Z-RNA binding thus upregulating IFN-stimulated genes [70]. Another likely pathogenic variant rs2076754 (c.C1295T: p.A432V) in the NOD2 gene associated with Blau syndrome/ Crohn's disease does not have any significant difference in comparison with the wild type [71]. However, it has a low allele frequency of $\leq 0.02 \%$ in the global population dataset, and also the odds of occurring in Crohn's disease patients is significantly higher than the controls [72]. Another nonsynonymous likely pathogenic mutation (c.G1390T: p.G464W) rs104895492 in the NOD2 gene has a low allele frequency of $0.003 \%$ in the global population. A reporter assay revealed this mutation to cause hyperactivity of NOD2-mediated NF-KB signaling in the absence of ligands [73]. The variant segregates with the disease in the mother and daughter, both were affected with Blau syndrome [74]. Another likely pathogenic nonsynonymous variant rs104895440 (c.C2137T: p.R713C) in the NOD2 gene has very low allele frequency of $<0.003 \%$ in the global population datasets. In vitro functional studies of the variant revealed major impairment of the peptidoglycan-induced response [71]. A nonsynonymous mutation (c.C1054T: p.R352C) in the NLRP12 gene found in two patients suffering from familial cold autoinflammatory syndrome (FCAS) of different ancestries. Functional study of the mutation revealed the increase in the speck formation as well as activation 
of the caspase 1 signaling in comparison with the wild type [11]. A likely pathogenic nonsynonymous variant rs200731780 (c.452G>A: p.R151Q) in the CARD14 gene associated with CARD14-mediated pustular psoriasis (CAMPS) that has been predicted as benign by computational prediction tools. However, in vitro functional studies of p.R151Q have shown a significant increase (i.e., 18 folds in the NF-KB activation that leads to CAMPS) $[75,76]$. It has a very low allele frequency of $<0.03 \%$ in the global population datasets (i.e., 1000 Genome Project, gnomAD, and Esp6500). Another likely pathogenic variant rs116107386 (c.11T>G: p.F4C) in the AP1S3 gene associated with pustular psoriasis has a frequency of $\sim 1 \%$ in the global population. However, in transfection studies in HEK293 cells, it showed a significant decrease of the mutant protein in comparison with the wild type that leads to marked inhibition of downstream signaling. The allele frequency was also found to be significantly higher in the patient than controls and falls in functionally important domains [77].

In this study, we have prioritized six causal founder variants. This includes rs28934897, rs148755083, rs202134424-T, rs202134424-G, rs200930463, and rs78635798 in the $M V K, I L 36 R N, A D A 2, A D A 2$, $A D A 2$, and $R N A S E H 2 C$ genes, respectively. The variant (rs28934897) in the $M V K$ gene is harbored in seven individuals of the IndiGen dataset in heterozygous state. On performing haplotype ancestry analysis, we found six out of seven individuals had admixed European ancestry. The occurrence of the European haplotype at the founder variant in the Indian population could be due to the invasion or migration of the Europeans in India [78]. The founder Dutch mutation p.V377I (rs28934897) along with the splicing mutation c.226+2delT in the $M V K$ gene in trans compound heterozygous state was found to be more common in the Indian population than reported [48]. An intronic variant c. $115+6 \mathrm{~T}>\mathrm{C}$ (rs148755083) in the gene IL36RN implicated in GPP was predominantly identified and considered as a founder variant in the East Asian population [50, 52-54]. We have identified this variant in two IndiGen individuals belonging to the Eastern part of India and was found to have East Asian ancestry. Since it has high frequency in the Eastern parts of India, variants could be screened, and the government could take proactive measures. We have also identified three variants p.G47V (rs202134424-T), p.G47V (rs202134424-G), and G47R (rs200930463) in the $A D A 2$ gene implicated in DADA2 known to be founder with high frequency in the Georgian Jewish population [57]. In IndiGen, out of the five individuals harboring these variants, three of them had European admixture, one East Asian, and one South Asian ancestry. A recent study performed in Indian DADA2 patients identified p.G47R as a founder variant in the Aggarwal/Jain community [59]. The Aggarwal community mainly resides in North India and is a descendant of the Indo-European migrants that had high frequency of the ADA2 causal variant $[59,79]$. The RNASEH2C variant p.R69W (rs78635798) implicated in AGS found in a single individual of IndiGen had South Asian haplotype ancestry. This variant was considered as a founder variant in the Asian populations [56].

In contrast with similar approaches towards understanding the genetic epidemiology of autoinflammatory diseases in other populations, three of the causal variants in the present analysis overlapped with our previous analysis of the Middle Eastern population [21]. Compared to the variant frequencies in the global populations, a number of disease alleles have frequencies in Indian population higher than global datasets including rs116107386, rs78635798, rs104895364, rs28934897, rs104895492, rs200930463, and rs202134424. Similarly, a number of variants have allele frequencies less than global populations like rs148755083 in IL36RN for pustular psoriasis. We surmise that the allele frequencies also correlate with the frequency of variants in clinical settings. For example, a recent case series of HIDS from our group suggests the clinical and genetic characteristics of patients. Incidentally, the prevalent variant rs148755083 with a founder effect was also the frequency variant identified in the case series [48].

The study has many caveats, the major being the dataset encompasses only a limited sample of cosmopolitan Indians, and therefore might not adequately cover smaller endogamous and ethnic groups. Secondly, the annotation of variants based on evidence from already proven cases, and therefore preclude novel and potentially pathogenic genetic variants.

\section{Conclusions}

The present analysis of genomes suggests that a number of autoinflammatory disease variants are prevalent in India. A subset of the variants were founders and were mainly descendants of different ancestry (i.e., European and East Asian due to migration or invasion in India). The causal founder autoinflammatory variant had high frequency with respect to their geographical regions or community. That could be considered a hotspot variant for the distinct population. The respective government could undertake the initiative and could perform the lowcost population screening so that it could provide better health care facilities to the population.

\section{Abbreviations}

VUS: Variant of uncertain significance; ACMG-AMP: American College of Medical Genetics and Genomics and Association of Molecular Pathology; FMF: Familial Mediterranean fever; CAPS: Cryopyrin-associated periodic syndrome; 
GME: Greater Middle East; IUIS: International Union of Immunological Societies; DADA2: Deficiency of adenosine deaminase 2; HIDS: Hyper IgD syndrome; GPP: Generalized pustular psoriasis; AGS: Aicardi-Goutières syndrome; FCAS: Familial cold autoinflammatory syndrome; CAMPS: CARD14-mediated pustular psoriasis.

\section{Supplementary Information}

The online version contains supplementary material available at https://doi. org/10.1186/s43141-021-00268-2.

Additional file 1: Supplementary Data 1. Detailed description of 28 ACMG-AMP guidelines for variant classification

Additional file 2: Supplementary Table 1. Autoinflammatory variants annotation and their classification according to the ACMG-AMP guidelines.

Additional file 3: Supplementary Table 2. IndiGen allele frequency comparison $p$-value $(<0.05)$ of autoinflammatory disorder pathogenic and likely pathogenic variants with the global databases included gnomAD V3, 1000 Genome Project, Esp6500, GME, and Qatar with their subpopulation. AMI: Amish, EUR (Fin): European (Finnish), EUR (Non Fin): European (Non Finnish), AFR: African, ASJ: Ashkenazi Jewish, EAS: East Asian, SAS: South Asian, AMR: American, BED Bedouin, SAF Sub-Saharan African, EUR European, SOU South Asian, APY African Pygmy, ARA ARAB, PER Persian, NWA: Northwest Africa, NEA: Northeast Africa, TP: Turkish Peninsula, SD: Syrian Desert, AP:Arabian Peninsula, and PP: Persia and Pakistan, NA not applicable. Significant values are marked with * and cells colored in red.

\section{Acknowledgments}

The authors acknowledge the volunteers who participated in this study. Authors acknowledge Mukta Poojary and Vishu Gupta for constructive suggestions and Sr. B. Velangini, Principal, St. Pious X Degree \& PG College for Women, Hyderabad for encouragement.

\section{Authors' contributions}

AJ was involved in the ACMG analysis, manuscript writing, and editing. RCB, AM, MI, VSen, MKD, SSharma, and SSiwach were involved in the whole genome sequencing. AJ, KV, DS, MR, and BJ were involved in the analysis of the whole genome sequencing. VScaria and SSB designed the study and were involved in the manuscript editing. The other authors were involved in the collection of the samples and formal analysis. The authors read and approved the final manuscript.

\section{Funding}

The study was funded by the Council of Scientific and Industrial Research (CSIR), India (MLP1809, MLP2001), CSIR fellowship (to A.J., M.K.D., B.J., A.B., S.M., R.Y.), Intel Research Fellowship (to D.S.), and UGC fellowship (to V.G., S.G.). Funding for open access charge: Council of Scientific and Industrial Research, India (MLP1809, MLP2001).

\section{Availability of data and materials}

All data generated or analyzed during this study are included in this published article (and its Supplementary information files).

\section{Declarations}

Ethics approval and consent to participate

This study was approved by the Institutional Human Ethics Committee (IHEC) (ethics approval no. CSIR-IGIB/IHEC/2018-19 Dt 21/02/2019) of CSIR-Institute of Genomics and Integrative Biology. The participants were explained about the informed consent process as per the approved IHEC guidelines and obtained written consent.

\section{Consent for publication}

A well informed consent has been obtained regarding publication from the participants.

\section{Competing interests}

The authors declare that they have no competing interests.

\section{Author details}

${ }^{1}$ CSIR-Institute of Genomics and Integrative Biology, New Delhi 110025, India. ${ }^{2}$ Academy of Scientific and Innovative Research (AcSIR), Ghaziabad, Uttar Pradesh 201002, India. ${ }^{3}$ Department of Zoology, RTM Nagpur University, Nagpur, Maharashtra 440033, India. ${ }^{4}$ Department of Zoology, Shri Mathuradas Mohota College of Science, Nagpur, Maharashtra 440009, India. ${ }^{5}$ Department of Anthropology, Vinoba Bhave University, Hazaribag, Jharkhand 825301, India. ${ }^{6}$ Department of Biotechnology, Vinoba Bhave University, Hazaribag, Jharkhand 825301, India. ${ }^{7}$ Department of Biochemistry, Dr. Kongu Science and Art College, Erode, Tamil Nadu 638107, India. ${ }^{8}$ Thalassemia and Sickle Cell Society, Hyderabad, Telangana 500052, India. ${ }^{9}$ Department of Biotechnology, Mizoram University, Aizawl, Mizoram 796004, India. ${ }^{10}$ Department of Pathology, Civil Hospital Aizawl, Mizoram 796001, India. ${ }^{11}$ TVMC, Tirunelveli Medical College, Tirunelveli, Tamil Nadu 627011, India. ${ }^{12}$ Department of Microbiology, St.Pious X Degree \& PG College for Women, Hyderabad, Telangana 500076, India.

${ }^{13}$ Department of Microbiology, The Maharaja Sayajirao University of Baroda, Vadodara, Gujarat 390002, India. ${ }^{14}$ Department of Dermatology, Venereology and Leprology, Regional Institute of Medical Sciences, Imphal, Manipur 795004, India. ${ }^{15}$ CSIR- North East Institute of Science and Technology, Jorhat, Assam 785006, India. ${ }^{16}$ Department of Dermatology, Dr. D.Y. Patil Medical College, Pune, Maharashtra 411018, India.

Received: 26 July 2021 Accepted: 13 October 2021

Published online: 14 December 2021

\section{References}

1. Rowczenio D, Shinar Y, Ceccherini I, Sheils K, Van Gijn M, Patton SJ et al (2019) Current practices for the genetic diagnosis of autoinflammatory diseases: results of a European Molecular Genetics Quality Network Survey. Eur J Hum Genet 27:1502-1508

2. Hausmann JS, Lomax KG, Shapiro A, Durrant K (2018) The patient journey to diagnosis and treatment of autoinflammatory diseases. Orphanet J Rare Dis 13:156

3. Federici S, Sormani MP, Ozen S, Lachmann HJ, Amaryan G, Woo P et al (2015) Evidence-based provisional clinical classification criteria for autoinflammatory periodic fevers. Ann Rheum Dis 74:799-805

4. Jia T, Zheng Y, Feng C, Yang T, Geng S (2020) A Chinese case of NakajoNishimura syndrome with novel compound heterozygous mutations of the PSMB8 gene. BMC Med Genet 21:126

5. Jia X, Shi N, Feng Y, Li Y, Tan J, Xu F et al (2020) Identification of 67 pleiotropic genes associated with seven autoimmune/autoinflammatory diseases using multivariate statistical analysis. Front Immunol 11:30

6. Chear CT, Nallusamy R, Canna SW, Chan KC, Baharin MF, Hishamshah M et al (2020) A novel de novo NLRC4 mutation reinforces the likely pathogenicity of specific LRR domain mutation. Clin Immunol 211:108328

7. Tao P, Sun J, Wu Z, Wang S, Wang J, Li W et al (2020) A dominant autoinflammatory disease caused by non-cleavable variants of RIPK1. Nature 577:109-114

8. Touitou I, Lesage S, McDermott M, Cuisset L, Hoffman H, Dode C et al (2004) Infevers: an evolving mutation database for auto-inflammatory syndromes. Hum Mutat 24:194-198

9. Hashkes PJ, Laxer RM, Simon A (2019) Textbook of autoinflammation. Springer, Cham. https://doi.org/10.1007/978-3-319-98605-0

10. Rusmini M, Federici S, Caroli F, Grossi A, Baldi M, Obici L et al (2016) Next-generation sequencing and its initial applications for molecular diagnosis of systemic auto-inflammatory diseases. Ann Rheum Dis 75:1550-1557

11. Jéru I, Le Borgne G, Cochet E, Hayrapetyan H, Duquesnoy P, Grateau G et al (2011) Identification and functional consequences of a recurrent NLRP12 missense mutation in periodic fever syndromes. Arthritis Rheum 63:1459-1464

12. Hoffman HM, Mueller JL, Broide DH, Wanderer AA, Kolodner RD (2001) Mutation of a new gene encoding a putative pyrin-like protein causes familial cold autoinflammatory syndrome and Muckle-Wells syndrome. Nat Genet 29:301-305 
13. Tsimaratos M, Kone-Paut I, Divry P, Philip N, Chabrol B (2001) Mevalonic aciduria and hyper-lgD syndrome: two sides of the same coin? J Inherit Metab Dis 24:413-414

14. Schnappauf O, Aksentijevich I (2019) Current and future advances in genetic testing in systemic autoinflammatory diseases. Rheumatology 58:vi44-vi55

15. Biesecker LG, Shianna KV, Mullikin JC (2011) Exome sequencing: the expert view. Genome Biol 12:128

16. Chou J, Ohsumi TK, Geha RS (2012) Use of whole exome and genome sequencing in the identification of genetic causes of primary immunodeficiencies. Curr Opin Allergy Clin Immunol 12:623-628

17. Sheppard S, Biswas S, Li MH, Jayaraman V, Slack I, Romasko EJ et al (2018) Utility and limitations of exome sequencing as a genetic diagnostic tool for children with hearing loss. Genet Med 20:1663-1676

18. Belkadi A, Bolze A, Itan Y, Cobat A, Vincent QB, Antipenko A et al (2015) Whole-genome sequencing is more powerful than whole-exome sequencing for detecting exome variants. Proc Natl Acad Sci U S A 112:5473-5478

19. Jain A, Govindaraj GM, Edavazhippurath A, Faisal N, Bhoyar RC, Gupta V et al (2021) Whole genome sequencing identifies novel structural variant in a large Indian family affected with X-linked agammaglobulinemia. PLoS One 16:e0254407

20. Thaventhiran JED, Lango Allen H, Burren OS, Rae W, Greene D, Staples E et al (2020) Whole-genome sequencing of a sporadic primary immunodeficiency cohort. Nature 583:90-95

21. Sharma P, Jain A, Scaria V (2021) Genetic landscape of rare autoinflammatory disease variants in Qatar and Middle Eastern populations through the integration of whole-genome and exome datasets. Front Genet $12: 631340$

22. Kumar BS, Kumar PS, Sowgandhi N, Prajwal BM, Mohan A, Sarma KVS et al (2016) Identification of novel mutations in CD2BP1 gene in clinically proven rheumatoid arthritis patients of South India. Eur J Med Genet 59:404-412

23. Jain A, Bhoyar RC, Pandhare K, Mishra A, Sharma D, Imran M et al (2021) IndiGenomes: a comprehensive resource of genetic variants from over 1000 Indian genomes. Nucleic Acids Res 49:D1225-D1232

24. Wang K, Li M, Hakonarson H (2010) ANNOVAR: functional annotation of genetic variants from high-throughput sequencing data. Nucleic Acids Res 38:e164

25. Pruitt KD, Tatusova T, Maglott DR (2007) NCBI reference sequences (RefSeq): a curated non-redundant sequence database of genomes, transcripts and proteins. Nucleic Acids Res 35:D61-D65

26. Sherry ST, Ward MH, Kholodov M, Baker J, Phan L, Smigielski EM et al (2001) dbSNP: the NCBI database of genetic variation. Nucleic Acids Res 29:308-311

27. Liu X, Wu C, Li C, Boerwinkle E (2016) dbNSFP v3.0: a one-stop database of functional predictions and annotations for human nonsynonymous and splice-site SNVs. Hum Mutat 37:235-241

28. Karczewski KJ, Francioli LC, Tiao G, Cummings BB, Alföldi J, Wang Q et al (2020) The mutational constraint spectrum quantified from variation in 141,456 humans. Nature 581:434-443

29. 1000 Genomes Project Consortium, Auton A, Brooks LD, Durbin RM Garrison EP, Kang HM et al (2015) A global reference for human genetic variation. Nature 526:68-74

30. Exome Variant Server n.d. http://evs.gs.washington.edu/EVS/ (Accessed 28 Mar 2021)

31. Scott EM, Halees A, Itan Y, Spencer EG, He Y, Azab MA et al (2016) Characterization of Greater Middle Eastern genetic variation for enhanced disease gene discovery. Nat Genet 48:1071-1076

32. Landrum MJ, Lee JM, Benson M, Brown GR, Chao C, Chitipiralla S et al (2018) ClinVar: improving access to variant interpretations and supporting evidence. Nucleic Acids Res 46:D1062-D1067

33. Tangye SG, Al-Herz W, Bousfiha A, Chatila T, Cunningham-Rundles C, Etzioni A et al (2020) Human inborn errors of immunity: 2019 update on the classification from the International Union of Immunological Societies Expert Committee. J Clin Immunol 40:24-64

34. Milhavet F, Cuisset L, Hoffman HM, Slim R, El-Shanti H, Aksentijevich I et al (2008) The Infevers autoinflammatory mutation online registry: update with new genes and functions. Hum Mutat 29:803-808

35. Infevers http://fmf.igh.cnrs.fr/ISSAID/infevers/index.php (Accessed 28 Mar 2021).
36. Richards S, Aziz N, Bale S, Bick D, Das S, Gastier-Foster J et al (2015) Standards and guidelines for the interpretation of sequence variants: a joint consensus recommendation of the American College of Medical Genetics and Genomics and the Association for Molecular Pathology. Genet Med 17:405-424

37. Genetic Variant Interpretation Tool https://www.medschool.umaryland.edu/ Genetic_Variant_Interpretation_Tool1.html/ (Accessed 28 Mar 2021).

38. Exome Variant Server http://evs.gs.washington.edu/EVS/ (Accessed 28 Mar 2021).

39. Lawson DJ, Hellenthal G, Myers S, Falush D (2012) Inference of population structure using dense haplotype data. PLoS Genet 8:e1002453

40. Delaneau O, Coulonges C, Zagury J-F (2008) Shape-IT: new rapid and accurate algorithm for haplotype inference. BMC Bioinformatics 9:540

41. Koshy R, Sivadas A, Scaria V (2018) Genetic epidemiology of familial Mediterranean fever through integrative analysis of whole genome and exome sequences from Middle East and North Africa. Clin Genet 93:92-102

42. Cush JJ (2013) Autoinflammatory syndromes. Dermatol Clin 31:471-480

43. Cuisset L, Jeru I, Dumont B, Fabre A, Cochet E, Le Bozec J et al (2011) Mutations in the autoinflammatory cryopyrin-associated periodic syndrome gene: epidemiological study and lessons from eight years of genetic analysis in France. Ann Rheum Dis 70:495-499

44. Sarkisian T, Ajrapetian H, Beglarian A, Shahsuvarian G, Egiazarian A (2008). Familial Mediterranean fever in Armenian population. Georgian Med News 105-11.

45. Moradian MM, Sarkisian T, Ajrapetyan H, Avanesian N (2010) Genotype-phenotype studies in a large cohort of Armenian patients with familial Mediterranean fever suggest clinical disease with heterozygous MEFV mutations. J Hum Genet 55:389-393

46. Houten SM, van Woerden CS, Wijburg FA, Wanders RJA, Waterham HR (2003) Carrier frequency of the V377I (1129G >A) MVK mutation, associated with Hyper-lgD and periodic fever syndrome, in the Netherlands. Eur J Hum Genet 11:196-200

47. Sandhya P, Vellarikkal SK, Nair A, Ravi R, Mathew J, Jayarajan R et al (2017) Egyptian tale from India: application of whole-exome sequencing in diagnosis of atypical familial Mediterranean fever. Int J Rheum Dis 20:1770-1775

48. Govindaraj GM, Jain A, Peethambaran G, Bhoyar RC, Vellarikkal SK, Ganapati A et al (2020) Spectrum of clinical features and genetic variants in mevalonate kinase (MVK) gene of South Indian families suffering from hyperimmunoglobulin D syndrome. PLoS One 15:e0237999

49. Körber A, Mössner R, Renner R, Sticht H, Wilsmann-Theis D, Schulz P et al (2013) Mutations in IL36RN in patients with generalized pustular psoriasis. J Invest Dermatol 133:2634-2637

50. Setta-Kaffetzi N, Navarini AA, Patel VM, Pullabhatla V, Pink AE, Choon S-E et al (2013) Rare pathogenic variants in IL36RN underlie a spectrum of psoriasisassociated pustular phenotypes. J Invest Dermatol 133:1366-1369

51. Onoufriadis A, Simpson MA, Pink AE, Di Meglio P, Smith CH, Pullabhatla $\checkmark$ et al (2011) Mutations in IL36RN/IL1F5 are associated with the severe episodic inflammatory skin disease known as generalized pustular psoriasis. Am J Hum Genet 89:432-437

52. Li M, Han J, Lu Z, Li H, Zhu K, Cheng R et al (2013) Prevalent and rare mutations in IL-36RN gene in Chinese patients with generalized pustular psoriasis and psoriasis vulgaris. J Invest Dermatol 133:2637-2639

53. Farooq M, Nakai H, Fujimoto A, Fujikawa H, Matsuyama A, Kariya N et al (2013) Mutation analysis of the IL36RN gene in 14 Japanese patients with generalized pustular psoriasis. Hum Mutat 34:176-183

54. Sugiura K, Takemoto A, Yamaguchi M, Takahashi H, Shoda Y, Mitsuma T et al (2013) The majority of generalized pustular psoriasis without psoriasis vulgaris is caused by deficiency of interleukin-36 receptor antagonist. J Invest Dermatol 133:2514-2521

55. Reijns MAM, Bubeck D, Gibson LCD, Graham SC, Baillie GS, Jones EY et al (2011) The structure of the human RNase H2 complex defines key interaction interfaces relevant to enzyme function and human disease. J Biol Chem 286:10530-10539

56. Vogt J, Agrawal S, Ibrahim Z, Southwood TR, Philip S, Macpherson L et al (2013) Striking intrafamilial phenotypic variability in Aicardi-Goutières syndrome associated with the recurrent Asian founder mutation in RNASEH2C. Am J Med Genet A 161A:338-342

57. Navon Elkan P, Pierce SB, Segel R, Walsh T, Barash J, Padeh S et al (2014) Mutant adenosine deaminase 2 in a polyarteritis nodosa vasculopathy. $\mathrm{N}$ Engl J Med 370:921-931 
58. Zhou Q, Yang D, Ombrello AK, Zavialov AV, Toro C, Zavialov AV et al (2014) Early-onset stroke and vasculopathy associated with mutations in ADA2. N Engl J Med 370:911-920

59. Sharma A, Naidu G, Sharma V, Jha S, Dhooria A, Dhir V et al (2021) Deficiency of adenosine deaminase 2 in adults and children: experience from India. Arthritis Rheumatol 73:276-285

60. Houten SM, Koster J, Romeijn GJ, Frenkel J, Di Rocco M, Caruso U et al (2001) Organization of the mevalonate kinase (MVK) gene and identification of novel mutations causing mevalonic aciduria and hyperimmunoglobulinaemia D and periodic fever syndrome. Eur J Hum Genet 9:253-259

61. Drenth JP, Cuisset L, Grateau G, Vasseur C, van de Velde-Visser SD, de Jong JG et al (1999) Mutations in the gene encoding mevalonate kinase cause hyper-IgD and periodic fever syndrome. International Hyper-lgD Study Group. Nat Genet 22:178-181

62. Houten SM, Kuis W, Duran M, de Koning TJ, van Royen-Kerkhof A, Romeijn GJ et al (1999) Mutations in MVK, encoding mevalonate kinase, cause hyperimmunoglobulinaemia D and periodic fever syndrome. Nat Genet 22:175-177

63. Cuisset L, Drenth JP, Simon A, Vincent MF, van der Velde VS, van der Meer JW et al (2001) Molecular analysis of MVK mutations and enzymatic activity in hyper-IgD and periodic fever syndrome. Eur J Hum Genet 9:260-266

64. Tanaka T, Yoshioka K, Nishikomori R, Sakai H, Abe J, Yamashita Y et al (2019) National survey of Japanese patients with mevalonate kinase deficiency reveals distinctive genetic and clinical characteristics. Mod Rheumatol 29:181-187

65. Galeotti C, Meinzer U, Quartier P, Rossi-Semerano L, Bader-Meunier B, Pillet $P$ et al (2012) Efficacy of interleukin-1-targeting drugs in mevalonate kinase deficiency. Rheumatology 51:1855-1859

66. Mandey SHL, Schneiders MS, Koster J, Waterham HR (2006) Mutational spectrum and genotype-phenotype correlations in mevalonate kinase deficiency. Hum Mutat 27:796-802

67. Çakan M, Aktay-Ayaz N, Keskindemirci G, Karadağ ŞG (2017) Two cases of periodic fever syndrome with coexistent mevalonate kinase and Mediterranean fever gene mutations. Turk J Pediatr 59:467-470

68. Rice Gl, Kitabayashi N, Barth M, Briggs TA, Burton ACE, Carpanelli ML et al (2017) Genetic, phenotypic, and interferon biomarker status in ADAR1related neurological disease. Neuropediatrics 48:166-184

69. Livingston JH, Lin J-P, Dale RC, Gill D, Brogan P, Munnich A et al (2014) A type I interferon signature identifies bilateral striatal necrosis due to mutations in ADAR1. J Med Genet 51:76-82

70. Rice Gl, Kasher PR, Forte GMA, Mannion NM, Greenwood SM, Szynkiewicz M et al (2012) Mutations in ADAR1 cause Aicardi-Goutières syndrome associated with a type I interferon signature. Nat Genet 44:1243-1248

71. Chamaillard M, Philpott D, Girardin SE, Zouali H, Lesage S, Chareyre F et al (2003) Gene-environment interaction modulated by allelic heterogeneity in inflammatory diseases. Proc Natl Acad Sci U S A 100:3455-3460

72. Naderi N, Farnood A, Habibi M, Zojaji H, Balaii H, Firouzi F et al (2011) NOD2 exonic variations in Iranian Crohn's disease patients. Int J Colorectal Dis 26:775-781

73. Parkhouse R, Boyle JP, Monie TP (2014) Blau syndrome polymorphisms in NOD2 identify nucleotide hydrolysis and helical domain 1 as signalling regulators. FEBS Lett 588:3382-3389

74. Khubchandani RP, Hasija R, Touitou I, Khemani C, Wouters CH, Rose CD (2012) Blau arteritis resembling Takayasu disease with a novel NOD2 mutation. J Rheumatol 39:1888-1892

75. Israel L, Mellett M (2018) Clinical and genetic heterogeneity of mutations in psoriatic skin disease. Front Immunol 9:2239

76. Ammar M, Jordan CT, Cao L, Lim E, Bouchlaka Souissi C, Jrad A et al (2016) CARD14 alterations in Tunisian patients with psoriasis and further characterization in European cohorts. Br J Dermatol 174:330-337

77. Setta-Kaffetzi N, Simpson MA, Navarini AA, Patel VM, Lu H-C, Allen MH et al (2014) AP1S3 mutations are associated with pustular psoriasis and impaired Toll-like receptor 3 trafficking. Am J Hum Genet 94:790-797

78. Bamshad M, Kivisild T, Watkins WS, Dixon ME, Ricker CE, Rao BB et al (2001) Genetic evidence on the origins of Indian caste populations. Genome Res 11:994-1004

79. Zhao Z, Khan F, Borkar M, Herrera R, Agrawal S (2009) Presence of three different paternal lineages among North Indians: a study of $560 \mathrm{Y}$ chromosomes. Ann Hum Biol 36:46-59

\section{Publisher's Note}

Springer Nature remains neutral with regard to jurisdictional claims in published maps and institutional affiliations.

\section{Submit your manuscript to a SpringerOpen ${ }^{\circ}$ journal and benefit from:}

- Convenient online submission

- Rigorous peer review

- Open access: articles freely available online

- High visibility within the field

- Retaining the copyright to your article

Submit your next manuscript at $\boldsymbol{\nabla}$ springeropen.com 